\title{
Consumer Attitudes Towards Environmental Concerns of Meat Consumption: A Systematic Review
}

\author{
Ruben Sanchez-Sabate ${ }^{1, *(1)}$ and Joan Sabaté ${ }^{2}$ (I) \\ 1 Centro de Excelencia en Psicología Económica y del Consumo (CEPEC), Núcleo Científico y Tecnológico en \\ Ciencias Sociales y Humanidades, Universidad de La Frontera, Temuco 4811230, Chile \\ 2 School of Public Health. Loma Linda University, Loma Linda, CA 92350, USA; jsabate@llu.edu \\ * Correspondence: ruben.sanchez@ufrontera.cl
}

Received: 1 March 2019; Accepted: 29 March 2019; Published: 5 April 2019

check for updates

\begin{abstract}
Meat consumption is a major contributor to global warming. Given the worldwide growing demand of meat, and the severe impact of meat production on the planet, reducing animal protein consumption is a matter of food security and public health. Changing consumer food behavior is a challenge. Taste preferences, culinary traditions and social norms factor into food choices. Since behavioral change cannot occur without the subject's positive attitude based on reasons and motivations, a total of 34 papers on consumer attitudes and behavior towards meat consumption in relation to environmental concerns were examined. The results show that consumers aware of the meat impact on the planet, willing to stop or significantly reduce meat consumption for environmental reasons, and who have already changed their meat intake for ecological concerns are a small minority. However, environmental motives are already appealing significant proportions of Westerners to adopt certain meat curtailment strategies. Those who limit meat intake for environmental reasons are typically female, young, simply meat-reducer (not vegan/vegetarian), ecology-oriented, and would more likely live in Europe and Asia than in the U.S.
\end{abstract}

Keywords: consumer attitudes; meat avoiders; meat reducers; environmental concerns; global warming; climate change; sustainability; ecology; planetary health

\section{Introduction}

Worldwide demand for meat and other animal products is increasing due to rising incomes, growing populations and other sociocultural factors [1,2]. This trend is a global problem because meat production is a major responsible for global warming and environmental degradation [1,3-6]. The livestock industry pollutes freshwater with antibiotics, hormones and chemical substances among others, depletes freshwater availability, contributes to the loss of biodiversity, and is a major source of anthropogenic greenhouse gas emissions [1]. Consequently, finding ways to make diets more sustainable by reducing animal protein consumption has become a matter of food security and thus, a public health issue [7].

Changing consumer food behaviors is a challenge. They are the result of strongly held factors like taste preferences, culinary traditions and social norms [8]. Health behavior theorists have described the stages a person undergoes when trying to adopt healthy behaviors. They cite that behavioral change can only occur with the adoption of a positive attitude based on reasons and motivations [9]. It is therefore relevant to know if environmental reasons can prompt individuals to reduce or avoid meat consumption.

In Western societies, meat-based diets are the norm. Meat avoiders like vegans and vegetarians represent a small minority. For instance, in the United States and the United Kingdom, vegetarians 
account for significantly less than $5 \%$ of the population [10]. The motivations of converted vegans and vegetarians - those raised on a meat-based diet-have been described as non-static and related to health, economy, environment, society and culture, ethics and religion [11]. Vegetarians can be categorized in two large groups: health oriented and ethically motivated [12]. This is because the most prevalent motivations among vegetarians are health and animal welfare [13-17]. Environmental reasons, on the contrary, are important to a small fraction of vegetarians [11].

Another group of consumers to consider are those not ready to give up meat, but who have, or are willing to consider reducing meat consumption. These are known as meat-reducers or flexitarians. Contrary to vegans and vegetarians who have been studied for decades, meat-reducers have received scant attention [18].

The goal of this systematic review is to enhance our understanding of consumer attitudes on meat consumption in relation to environmental sustainability in order to support potential public health interventions oriented towards meat intake reduction. We looked into the three main stages of behavioral change process as proposed by Glanz et al.'s [9]: awareness (precontemplation), willingness (contemplation and preparation) and change (action, maintenance and termination). Having a general overview of the three stages should give public health professionals a general understanding of the role environmental reasons may play in the food eating behavior change process. Thus, this systematic review aims to answer the three following research questions: (1) Are people aware of the environmental impact of meat production and consumption? (2) Are people willing to stop or reduce meat consumption based on environmental concerns? and (3) Have ecological/environmental concerns been the motivation for people who have altered their meat consumption?

\section{Materials and Methods}

This systematic review was reported following the PRISMA (Preferred Reporting Items for Systematic Reviews and Meta-Analyses) guidelines [19] We performed a preliminary search in Google Scholar for articles that reported data on at least one of the following three topics: people's awareness of the environmental impact of meat production and consumption; people's willingness to stop or reduce meat consumption because of environmental concerns; and people who have already stopped or reduced meat consumption because of environmental reasons or motivations (diet change).

This initial search allowed us to identify a series of keywords that we later used to conduct a literature search of the Web of Science (WOS) Core Collection in March 2018. A separate query was conducted for each topic (awareness, willingness and diet change). Each query consisted of a series of search strings that combined no more than three terms each from one of the following categories: consumer related, meat related, and planet related. For example, one query looked like this: consumer attitudes AND meat AND climate change.

Thus, for "awareness" we used a series of search strings that combined the following terms: "consumer/people attitudes/perceptions" AND "meat" / "livestock" AND "climate change" / "GHG emissions"/"global near/2 warming"/"environment"/"water near/3 use"/"land near/3 use". Similar search strings were used for "willingness" and "change".

The screening process was completed by both authors independently to reduce bias. It comprised three stages for each one of the three topics considered. First, articles and abstracts were screened. Citations that met the eligibility criteria (Figure 1) were imported to the reference manager Zotero. Second, selected citations were read in full to make a final decision on their relevance for any of the three topics considered, and to locate new relevant articles that had not been found by the WOS search. Third, these first two steps were conducted for the new bibliography until no new eligible references were detected. The few articles considered pertinent by only one reviewer were included or discarded after a discussion between the two coauthors. The search for "awareness" yielded a total of 14 articles that met the eligibility criteria. The search for "willingness" yielded a total of 16 articles that met the eligibility criteria. And the search for "diet change" yielded a total of 17 articles that met the eligibility criteria. This systematic review rendered a total of 34 articles since some publications were relevant 
for more than one topic. Pertinent data from these articles was abstracted in tables with categories including: study design, sample characteristics, question or dependent variable and covariates effects, among other relevant information.

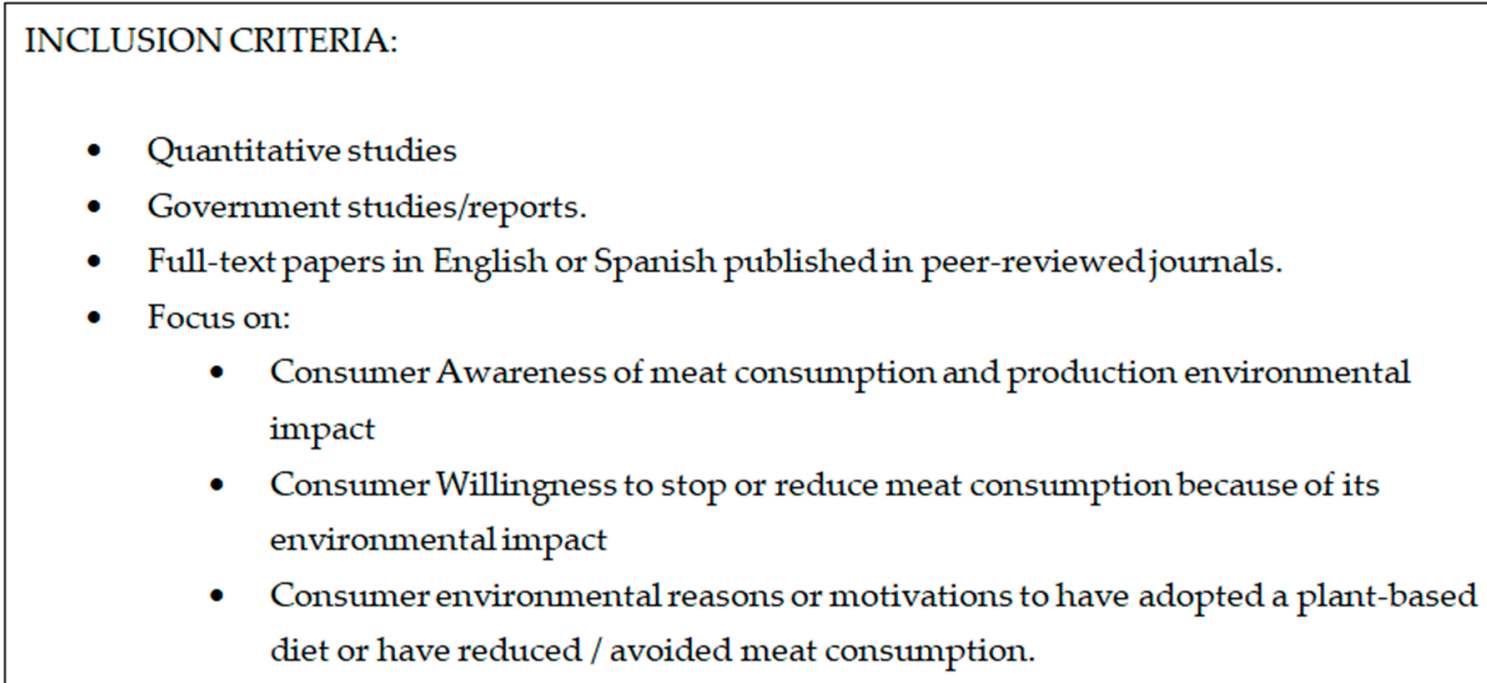

\section{EXCLUSION CRITERIA:}

- Qualitative studies

- Opinion papers, outlook, concept papers, books or book chapters.

- Not related to consumer attitudes or behavior (e.g. environmental impact of meat production).

- Studies that only consider one type of meat (e.g. beef, lamb, poultry...).

Figure 1. Eligibility criteria.

\section{Results}

\subsection{People Awareness of the Environmental Impact of Meat Production and Consumption}

The results from 14 articles that examined awareness of the negative impact meat production and consumption have on the environment are summarized in Table A1, presented in the Appendix A at the end of this dcocument. In short, the main findings are: (1) aware consumers are a minority; (2) consumers either underestimate or ignore the potential of either stopping or reducing meat production and consumption to reduce the anthropogenic impact on the environment; and (3) it is not clear for the consumer that a vegetarian diet is more environmental friendly than a diet including meat.

Consumer awareness of the meat environmental toll has been studied in Belgium, Finland, Germany, the Netherlands, Portugal and the United States using different methods. The percentages of aware participants ranged from $23 \%$ to $35 \%$ across studies [20-22]. One study in which subjects received prior information, the percentage jumped to $58 \%$ [23]. Another study required respondents to list concrete impacts of meat production on the planet: only $24 \%$ named "pollution" and $20 \%$ "erosion of natural resources" [24]. Another study showed a tendency toward a neutral opinion on the negative environmental impact of meat [25]. And regarding behaviors that damage the earth, one study showed that consumers rarely (less than 10\%) thought of "meat eating" [26].

Consumer estimation of meat production and consumption toll on the environment was studied in Australia, Belgium, the Netherlands, Switzerland, the United Kingdom, and the U.S. Only two studies specifically queried participants on meat production. Less than half $(38 \%)$ agreed that changing 
animal husbandry can counter climate change [20], but still its toll was underestimated relative to other activities like transport, even when prior information on meat and the environment was given [23]. All other studies focused on meat consumption reduction. Percentages of participants agreeing with it as a way to help the environment varied between $18 \%$ to $29 \%$ across studies [27-29]. Percentages of subjects that considered it an effective way to alleviate climate change varied from $5 \%$ to $64 \%$. This big range can be explained by different methodological and geographical factors across studies. Still, reducing meat consumption was usually considered the least or second least effective when compared to other options $[26,30,31]$. Still consistent with this finding, the only longitudinal study found by the reviewers showed that participants gave slightly higher effectiveness to meat reduction in the follow-up survey four years later [32]. Finally, it is not clear to consumers that a vegetarian diet is more environmentally friendly than a diet with meat [25].

Not all studies report on covariate effects. From those which do, the gender variable is the most frequent one. Women are more conscious about the negative impact meat has on the environment [22,25], and thus, they perceive a higher effectiveness in reducing meat consumption to alleviate climate change than men [26,30-33]. One study found that the only important covariates were the frequency of meat intake and already established concerns about the environment. As meat intake went up, the perceived effectiveness of meat reduction went down. But the subjects who held a strong belief in human causation of climate change assigned a positive association between eating less meat and helping the planet. Other covariates like age and level of education presented no correlations [30]. Another study also showed no correlations of awareness with age, but surprisingly, neither with gender nor with meat consumption frequency [28].

\subsection{People Willingness to Stop or Reduce Meat Consumption Because of Environmental Reasons or Motivations}

The results from 15 papers plus a European Union Report (EUR) that examined people willingness to stop or reduce meat consumption for environmental reasons are summarized in Table A2 (see Appendix B). The main findings are: (1) those motivated by ecological concerns to reduce meat intake are a minority, and (2) meat curtailment is among the least preferred personal options to counter climate change.

When no prior information on the meat environmental toll was given, participants from Finland, Germany, The Netherlands, Switzerland and the U.S. willing to stop or reduce meat consumption because of environmental reasons ranged from $12.8 \%$ to $25.5 \%$ [22,25,33]. Reducing meat intake was usually the least chosen option to curb climate change [26,30]. Belief in the negative impact of meat on the planet associated positively with willingness to change meat consumption in three studies $[26,30,33]$. One study also revealed a positive association between consciousness, understood as cognitive and affective awareness of the environmental toll of meat, with willingness to reduce meat consumption [22]. Another study that specifically distinguished between belief and actual knowledge on the effectiveness of meat reduction for climate change mitigation, showed that while belief was positively associated with willingness, knowledge was not [26]. Only one study explicitly reported that education and age were not related to willingness [33].

Eight studies conducted throughout Belgium, Germany, The Netherlands, Portugal, Sweden and the U.S., and the EUR did provide information to the participants connecting meat production and consumption with the environment before the data collection. The results show disparate percentages of people willing or maybe willing to reduce meat consumption for environmental reasons. If simply asked for their willingness to make such a dietary change, participants "certainly willing" were a small minority (5-18\%), while those "maybe willing" were $41 \%$ [20,25]. Regarding agreement with certain direct meat curtailment strategies, percentages varied widely $(15-60 \%)$ depending on the strategy considered. Meat substitution for vegetables was significantly less popular than meat reduction, but the latter was still among the least preferred options unless compared with eating insects or meat substitutes [21,23,24]. In one study, participants did not find altering meat consumption easy to do [34]. 
The EUR [35] reported that about $50 \%$ of Europeans would be willing to replace most of the meat they eat with vegetables, and $80 \%$ of them would be willing to eat less meat but of certified origin. Considering some countries separately, the UK, the Netherlands, Denmark, Finland and Belgium present lower percentages of people willing to replace meat with vegetables (29-49\%) and of people willing to consume less meat but of certified origin (62-73\%) than countries like Portugal, Spain, Italy and Romania in which percentages range from $53 \%$ to $69 \%$ and $83 \%$ to $89 \%$ respectively.

Covariate effects are similar to those presented in the awareness section. Being female is usually a strong predictor of willingness to decrease meat consumption or choose meat-free menus $[21,29,31,35,36]$. Meat consumption frequency and positive attitudes to meat are negatively associated with willingness to eat it less $[21,24,31,37]$. Ethnicity and culture can strongly influence willingness. Turks living in the Netherlands were less willing to alter meat consumption than Chinese and Native Dutch [36]. Mediterranean Europeans responded more positively to replacing most of the meat with vegetables (56\% average) and to reduce meat consumption $(86 \%)$ than Northern Europeans (46\% and $80 \%$, respectively) [38]. Regarding income, one study presented a negative association between affluence and willingness [38]. Age and education, on the contrary, had in general no influence [31].

Finally, the effect of information on meat and the environment on willingness is less clear. In one study, it could be seen that prior information increased the percentage of people willing to eat less meat from $12 \%$ to $18 \%$ [25]. In two other studies, information did not alter the number of participants willing to choose meals with less or no meat [21,29]. However, one study reported that participants concerned for the environment and/or already aware before the experiment about the negative impact of meat, were more likely to support meat curtailment strategies [21]. Still, another study found that pro-environmental beliefs had no significant predictive value [29]. In any case, it should be kept in mind that each study provided participants with different types, degrees, and formats of information on the meat environmental toll and thus, generalizing results is not recommendable.

\subsection{Meat Consumption Changes for Environmental Reasons}

The results from 17 articles that examined motivations for limiting meat consumption are summarized in Table A3 (Appendix C). The main findings show that those who have already adopted a meatless diet or have already reduced its consumption are: (1) a small minority among samples from the general population, and a significantly bigger one among certain population groups; and (2) female, most likely young, partial meat limiters and reside in Europe.

The studies reviewed referred to people who follow a low or no-animal product diet in two different ways: (1) vegans and vegetarians; and (2) "meat avoiders", "animal product limiters", and similar expressions. This fact directly affected the wording of questions and sentences that participants had to answer or rate. Thus, some studies looked for reasons for being "vegan", "vegetarian" or something similar like "semi-vegetarian", while other studies searched for reasons for "avoiding meat", "reducing meat consumption" or any other wording that means the curtailment of animal products consumption. It is necessary to bring attention to this point because veganism and vegetarianism are not only a diet choice but an identity [39]. Deciding to become a vegetarian is a much more complex process than simply opting for reducing or avoiding meat consumption, or even adopting a plant-based diet.

Studies that specifically asked for reasons or motives for being vegan/vegetarian were all conducted in the U.S. Those who indicated environmental concerns were few $(>3.2 \%)[39,40]$ in recent surveys with a general population of vegans/vegetarians. However, among specific population groups environmental vegan/vegetarians were significant minorities: $14 \%$ in the case of marathon runners [41], and 32.1\% in the case of women physicians surveyed two decades ago [42]. Other research conducted in the U.S. and Finland showed that vegans, vegetarians and semi-vegetarians tend to agree with and give a moderate importance to the protective benefits of a vegetarian diet towards the environment $[43,44]$. 
Only a few consumers (4-19\%) indicated environmental concerns for having reduced or avoided meat intake in studies conducted in Belgium, The Netherlands and the U.S. [23,45,46]. However, when specific population groups and certain meat curtailment strategies are considered the percentage of environmental meat reducers or avoiders increases. More than a $50 \%$ of a general population sample from The Netherlands reported to have "one meat-free day a week" and "smaller meat portions" at least once a month [47]. Other studies showed that meat avoiders/reducers gave a moderate importance to environmental concerns in their meat purchasing and consumption habits [48]. Those who considered ecology important were the $38.2 \%$ of a Dutch sample $[45,49,50]$. And $38.1 \%$ of university students from eleven Eurasian countries pointed to the environment as their major reason for meat avoidance [51].

Reported covariate effects across studies, and research on specific groups like vegans, portray those who limit meat consumption because of the environment as female, young, semi-vegetarian/meat reducer, ecology-oriented, and more likely living in Europe and Asia than in the U.S. Four studies that specifically asked participants to indicate their main reason for meat reduction or avoidance further reflect this profile $[39,40,49,51]$. Once more, women proved more likely to reduce meat intake because of the environment than men. This was true for Euromerican women [48,49], and for a multiethnic sample from The Netherlands [47,52]. Studies rarely reported age as a significant covariate. However, considering the one study that did [43], and the fact that this review found the highest percentage of meat avoiders because of the environment, in a survey of 3433 students attending different universities based in eleven Eurasian countries [51], it appears that young people may be the most motivated by ecology for already having reduced or stopped meat intake. The degree of involvement with food and sustainability, regardless of age, is another covariate that also correlated positively with environmental reasons for meat curtailment [46,47]. Ethnicity, as well, had a significant impact in one study conducted in The Netherlands [36].

Considering only studies published after 2010, vegans and meat limiters may be more likely to be influenced by environmental reasons than vegetarians. Samples from the U.S., Europe and Asia presented much lower percentages (9-21\%) of vegetarians that consider sustainability an important factor that shapes their diet than semi-vegetarians (30-49\%), light semi-vegetarians (34-44\%) or meat limiters in general $(41 \%)[48,49,51,52]$. Two studies carried out in the U.S. before the year 2000, add to this pattern: $60.7 \%$ of all types of meat limiters including vegetarians [45] and $32.1 \%$ of self-described vegetarians indicated ecological concerns as current reason for their dietary choices [42]. An older study in the UK also showed that vegans and meat reducers are more likely to be influenced by environmental reasons than vegetarians [53]. Opposite results to this pattern, meaning that vegetarians reported to be more influenced by ecological concerns than vegans and meat reducers, appeared to a certain extent, in a study conducted in Finland [44]. In any case, more evidence is needed in order to draw conclusions on differences between vegans, vegetarians and meat reducers. Finally, two recent surveys of vegans living in the U.S. yielded very low percentages (2-3.2\%) of consumers motivated by the environment $[39,40]$, adding country location as another significant variable to consider.

\section{Discussion}

The reduction of meat production and consumption would alleviate the anthropogenic impact on the environment [1]. Individual choices for diets low in meat and high in vegetables are urgently needed according to the latest scientific evidence [7]. Previous studies have identified two main motivations that prompt people in the West to become vegan or vegetarian: animal welfare and health $[14,16,45,54]$. Ecological concerns, however, are only relevant to a minority of them [11]. In addition to vegans and vegetarians, there are a significant number of consumers who limit meat consumption. Known as meat-reducers or flexitarians, few studies have explored their motivations for reducing meat intake [18].

Review of the main findings shows that, in the so-called developed countries, those aware of the meat impact on the planet, and those willing to alter their meat consumption for environmental reasons, are a small minority. This result is in line with a previous review of awareness and willingness only [55]. 
Regarding change, the present review shows that people who altered their meat consumption patterns because of the environment represent also a small minority of the studied samples. Within this minority of people aware, willing, or who have already changed, women are a clear majority. Considering in addition that the reduction of meat consumption tends to be among the least preferred strategies to alleviate climate change when compared to other non-food activities like driving less, it looks like environmental reasons are not a major motive for reducing meat intake for the general Western population.

Giving information on the environmental toll of meat production could be a promising strategy to increase awareness and willingness. Studies that provided participants with such information before the test showed significantly higher percentages of people aware and willing. However, there are two other factors that could very well explain such increases. First, social desirability, i.e., survey respondents' tendency to give answers they believe will be viewed favorably by researchers or other participants. The second factor, which applies only to the studies reviewed on willingness, has to do with their different designs. Percentages of people willing to alter meat consumption when prior information is given vary from $5 \%$ to $80 \%$ in the papers reviewed. Such significant disparity could be explained by studies variations in: (1) methodology; (2) the assessed behavioral action state: some studies measured "belief" while others "intention" or "willingness"; (3) the definition of target behavior (it is not the same to aim for a plant-based diet than for eating meat-free meals regularly) and (4) the time frame to adopt the favorable behavior: for instance, having a meat-free meal $x$ times per month or per week. Therefore, it remains unclear how beneficial the strategy of informing the consumer on the meat environmental toll will actually be for the reduction of its intake.

It is also necessary to pay attention to how the information on the meat impact on the environment is usually introduced. The papers reviewed present the environmental problem in a very rational and detached way. By this we mean that prior information given or questions addressed to participants are based on the common-sense supposition that the environment is separate from, and around, humans. As Lakoff [56] has argued, this is a false supposition because humans are an inseparable part of nature. Yet, this mode of thinking and understanding ("frame" in communication sciences parlance) is common in mass media and public policy communications [56], as well as how scientists word the questions they use and how study participants interpret them. Thus, it is necessary to explore how subjects would react to meat curtailment strategies when ecological concerns are presented to them in an emotional fashion. Research on this regard is promising as environmental messages that appealed to emotions and/or values reduced the intentions of participants to eat meat and affected their attitudes towards meat consumption $[34,57,58]$. However, research on the effects of emotional messages on people's attitudes and behaviors towards climate change in general has shown that fear-based appeals can backfire and lead to a decrease in participants' willingness to reduce their carbon footprints [59]. In addition, a longitudinal study conducted in the UK showed that levels of concern and motivation to behaviorally address climate change decrease as time passes from participants' exposure to climate change communications [60]. Therefore, more research on communication strategies to increase awareness and willingness to alter meat consumption among Westerners is needed.

Surprisingly, despite increased media attention in recent years to the environmental concerns linked to meat consumption, percentages of vegan, vegetarian and meat reducer participants who claim to follow such dietary patterns on environmental concerns have remained largely unchanged in studies conducted after 2010 compared to the few published before 2002 included in this review. This could be explained by the fact that scientific knowledge and even dietary recommendations for reducing meat consumption based on environmental reasons precede the time span (1987-2016) of the studies included in this systematic review [61]. Such knowledge evidently permeated to vegans and vegetarians long before the more recent mass media attention, probably because they have belief systems and/or sources of information outside the mainstream.

The studies reviewed have limitations that should be addressed in future research. The geographical limitation (the fact that the majority of studies were conducted in only a small 
number of countries of northern Europe and North America) is the most noticeable. The large survey carried out by the European Commission showed big differences in willingness between northern and southern European countries [35,38]. This gives reason to believe that research on awareness, willingness, and change regarding meat consumption in relation to planetary health can yield significantly different results when Mediterranean, Latin American, and the so-called developing countries are considered. Were this the case, such differences could be explained due to cultural and economic determinants.

There are also methodological limitations worth considering when designing future studies. The majority of the studies reviewed used convenience samples. Random samples are better in order to generalize results to the general populations. Another limitation is that we have found only one longitudinal study. Longitudinal studies could be of interest to identify the evolution of the influence environmental reasons may have on subjects throughout their lives. Cultural aspects may not have been sufficiently taken into account. One study noted large differences in willingness and diet change across ethnicities living in the same country [36]. Further research exploring willingness and change could benefit from an understanding of the cultural significance meat has in the culture/society to be studied. For this and the geographical limitation mentioned before, we consider the results of this systematic review hard to generalize cross-nationally.

Future research could incorporate covariates such as gastronomic and hedonistic dimensions of meat intake and people's cooking skills when examining willingness and change. Previous studies have already shown that people rarely want to give up meat for the pleasure it gives them $[27,62,63]$. Thus, it is probable that those who do not have the skills to cook palatable meat-free meals, may not reduce its consumption not because they do not want to, but because they do not know how to have an enjoyable food experience without meat. Another covariate to consider in future research is the participant's social networks. Since eating is a socially regulated behavior [64], such an important dietary change as altering meat consumption may be favored or impeded by, for instance, family and/or significant communities such as churches, vegetarian associations.

\section{Conclusions}

This systematic review reveals a lack of disposition by the general population in Western countries to stop eating meat on environmental reasons. Even for vegans/vegetarians, ecological concerns are more of another motive to further justify their dietary pattern than an original motivation to give up animal products altogether. However, the reviewed evidence also shows that environmental motives are already appealing to significant proportions of Western meat-eaters to adopt certain meat curtailment strategies like meat-free days. This appeal is more prevalent among women and people from certain cultures. Given that dietary habits are not static, and the fact that mass media attention to sustainable food systems and diets is increasing, it is feasible that ecological concerns become a trigger to at least minor reductions in meat consumption for a majority of the Western population, especially for those not motivated by health or animal welfare. Since a small reduction in meat intake among a large proportion of Westerners could mean a significant contribution to reducing the anthropogenic impact on the environment, mass media outlets, public health educators, nutritionists, policy makers, and the food industry may also consider environmental reasons to promote healthy and sustainable diets.

Author Contributions: Both authors contributed to the conceptualization of this systematic review. R.S.-S. wrote the original draft. Both authors reviewed and edited the original draft. Both authors have read and approved the final manuscript.

Funding: No funding received.

Conflicts of Interest: The authors declare no conflict of interest. 


\section{Appendix A}

Table A1. People's awareness of the environmental impact of meat production and consumption.

\begin{tabular}{|c|c|c|c|c|c|c|c|}
\hline \multicolumn{5}{|l|}{ Title } & \multicolumn{3}{|c|}{ Outcome Measure: Perceived Environmental Impact } \\
\hline $\begin{array}{l}\text { Author(s), } \\
\text { Year }\end{array}$ & $\begin{array}{l}\text { Design; Year } \\
\text { Data Collected }\end{array}$ & Country; Sample & $\begin{array}{l}\text { Main Research } \\
\text { Question }\end{array}$ & $\begin{array}{l}\text { Provided } \\
\text { Information Prior } \\
\text { to the Experiment }\end{array}$ & $\begin{array}{c}\text { Question or Dependent } \\
\text { Variable }\end{array}$ & Response or Finding & Effect of Covariates \\
\hline $\begin{array}{l}\text { Campbell- } \\
\text { Arvai, } 2015 \\
\text { [29] * }\end{array}$ & $\begin{array}{l}\text { Survey in } \\
\text { dining halls; } \\
\text { unspecified }\end{array}$ & $\begin{array}{l}\text { U.S.; undergraduate } \\
\text { students, convenience } \\
\text { sample, } N=320,46 \% \text { men }\end{array}$ & $\begin{array}{l}\text { Food-related } \\
\text { environmental } \\
\text { beliefs and } \\
\text { behaviors }\end{array}$ & No info & $\begin{array}{l}\text { (1) Eating less meat can } \\
\text { help the environment. } \\
\text { (2) Adopting a vegetarian } \\
\text { diet can help the } \\
\text { environment }\end{array}$ & $\begin{array}{l}\text { (1) } 29 \% \text { agree; } 20 \% \text { unsure; } \\
51 \% \text { disagree } \\
\text { (2) } 22 \% \text { agree; } 13 \% \text { unsure; } \\
65 \% \text { disagree Lowest level } \\
\text { of agreement compared } \\
\text { with other behaviors (e.g., } \\
\text { using less packaging, } \\
\text { grown locally) }\end{array}$ & n.a. \\
\hline $\begin{array}{l}\text { Clonan et al., } \\
2015[28]^{*}\end{array}$ & $\begin{array}{l}\text { Postal survey; } \\
2009\end{array}$ & $\begin{array}{l}\text { UK (Nottinghamshire); } \\
\text { random sample from } \\
\text { electoral registers, } \\
N=842,41 \% \text { men }\end{array}$ & $\begin{array}{l}\text { Meat consumption } \\
\text { attitudes and } \\
\text { sustainable meat } \\
\text { purchase }\end{array}$ & No info & $\begin{array}{l}\text { To help reduce the impact } \\
\text { of climate change, it is } \\
\text { better to eat less animal } \\
\text { foods (meat, dairy } \\
\text { products and eggs). }\end{array}$ & $\begin{array}{l}18 \% \text { agree } \\
46 \% \text { unsure } \\
36 \% \text { disagree }\end{array}$ & $\begin{array}{l}\text { Red and processed meat } \\
\text { intake frequency, } \\
\text { sustainable meat purchase } \\
\text { frequency, gender, age, SES } \\
\text { were not significant }\end{array}$ \\
\hline $\begin{array}{l}\text { Cordts et al., } \\
2014[25]^{*}\end{array}$ & $\begin{array}{l}\text { Online } \\
\text { experiment; } \\
2013\end{array}$ & $\begin{array}{l}\text { Germany; quota sample, } \\
N=590,52 \% \text { men }\end{array}$ & $\begin{array}{l}\text { Consumer } \\
\text { response to } \\
\text { negative } \\
\text { information on } \\
\text { meat consumption }\end{array}$ & $\begin{array}{l}\text { Variables } \\
\text { measured before } \\
\text { info provision } \\
\text { (experimental } \\
\text { manipulation) }\end{array}$ & $\begin{array}{l}\text { (1) Farming animals and } \\
\text { producing animal } \\
\text { products (e.g., milk or } \\
\text { meat) has a considerable } \\
\text { negative environmental } \\
\text { impact. } \\
\text { (2) A vegetarian diet is } \\
\text { more environmentally } \\
\text { friendly than a diet } \\
\text { including meat. }\end{array}$ & $\begin{array}{l}\text { (1) } \mathrm{M}=3.07, \mathrm{SD}=1.12 \\
(1=\text { do not agree at all to } \\
5=\text { fully agree) } \\
\text { (2) } \mathrm{M}=3.10, \mathrm{SD}=1.21 \\
(1=\text { do not agree at all to } \\
5=\text { fully agree) }\end{array}$ & $\begin{array}{l}\text { (1) Women agreed more } \\
\text { than men }(\mathrm{M}=3.19 ; \\
\mathrm{SD}=1.11 ; \mathrm{M}=2.95 ; \\
\mathrm{SD}=1.12 ; p \leq 0.01) \\
\text { (2) Women agreed more } \\
\text { than men }(\mathrm{M}=3.23 ; \\
\mathrm{SD}=1.19, \mathrm{M}=2.98 ; \\
\mathrm{SD}=1.21 ; p \leq 0.05)\end{array}$ \\
\hline
\end{tabular}


Table A1. Cont.

\begin{tabular}{|c|c|c|c|c|c|c|c|}
\hline \multicolumn{5}{|l|}{ Title } & \multicolumn{3}{|c|}{ Outcome Measure: Perceived Environmental Impact } \\
\hline $\begin{array}{l}\text { Author(s), } \\
\text { Year }\end{array}$ & $\begin{array}{l}\text { Design; Year } \\
\text { Data Collected }\end{array}$ & Country; Sample & $\begin{array}{l}\text { Main Research } \\
\text { Question }\end{array}$ & $\begin{array}{l}\text { Provided } \\
\text { Information Prior } \\
\text { to the Experiment }\end{array}$ & $\begin{array}{l}\text { Question or Dependent } \\
\text { Variable }\end{array}$ & Response or Finding & Effect of Covariates \\
\hline $\begin{array}{l}\text { De Boer et al., } \\
2016 \text { [30] }\end{array}$ & $\begin{array}{l}\text { Nation-wide } \\
\text { consumer } \\
\text { surveys; } 2014\end{array}$ & $\begin{array}{l}\text { Netherlands and the USA; } \\
\text { representative sample } \\
N=527 \text { (The Netherlands). } \\
\text { Weighted variables: gender, } \\
\text { age, level of education, } \\
\text { region, and a value-related } \\
\text { test score on } \\
\text { "mentality-environment". } \\
\text { (efficiency of the weighting } \\
89 \% \text {, effective sample } \\
\text { size } 478) N=556 \text { (USA). } \\
\text { Weighted variables: gender, } \\
\text { age, and level of education } \\
\text { (efficiency of the weighting } \\
90 \% \text {, effective sample } \\
\text { size 500) Total = } 1083\end{array}$ & $\begin{array}{l}\text { Consumer } \\
\text { awareness of meat } \\
\text { consumption } \\
\text { environmental } \\
\text { impact and their } \\
\text { willingness to } \\
\text { reduce meat } \\
\text { consumption, } \\
\text { among other } \\
\text { research questions. }\end{array}$ & $\begin{array}{l}\text { No prior info } \\
\text { given. }\end{array}$ & $\begin{array}{l}\text { "For each of the following } \\
\text { lifestyle- changes, please } \\
\text { let us know whether you } \\
\text { think this is an effective } \\
\text { way of combatting climate } \\
\text { change". The options, } \\
\text { which were presented in } \\
\text { randomized order, were: } \\
\text { "Eat less meat", "Buy } \\
\text { local, seasonal, } \\
\text { unprocessed foods (e.g., } \\
\text { by going to farmer's } \\
\text { markets)", "Buy (more) } \\
\text { organic foods", "Drive } \\
\text { less", "Save energy at } \\
\text { home (e.g., turning } \\
\text { thermostat down, using } \\
\text { saving bulbs, air-drying } \\
\text { laundry)", and "Install } \\
\text { solar panels on my } \\
\text { house". }\end{array}$ & $\begin{array}{l}\text { Dutch: "eating less meat" } \\
\text { option, second less effective } \\
12 \% \text { recognized the } \\
\text { outstanding effectiveness of } \\
\text { the less meat option in the } \\
\text { eyes of climate experts } 46 \% \\
\text { attributed effectiveness to } \\
\text { the "eating less meat" } \\
\text { option Americans: "eating } \\
\text { less meat" option, the least } \\
\text { effective } 6 \% \text { recognized the } \\
\text { outstanding effectiveness of } \\
\text { the less meat option in the } \\
\text { eyes of climate experts } \\
30 \% \text { attributed effectiveness } \\
\text { to the "eating less meat" } \\
\text { option }\end{array}$ & $\begin{array}{l}\text { Regular meat eaters } \\
\text { assigned lower effectiveness } \\
\text { ratings to the less meat and } \\
\text { the organic food option, but } \\
\text { not to the other options. } \\
\text { Belief in human causation } \\
\text { and personal importance } \\
\text { were associated with } \\
\text { assigning higher } \\
\text { effectiveness ratings to all } \\
\text { the options. } \\
\text { The pattern of profile results } \\
\text { remained unchanged when } \\
\text { gender, age, and level of } \\
\text { education were entered as } \\
\text { covariates. The analysis } \\
\text { revealed that these variables } \\
\text { had small effects on the } \\
\text { effectivity ratings. Females } \\
\text { gave slightly higher ratings } \\
\text { than males, especially to the } \\
\text { food-related options }\end{array}$ \\
\hline $\begin{array}{l}\text { Study } 1 \\
\text { de Boer, } \\
\text { Schösler, et al., } \\
2013 \text { [20] * }\end{array}$ & $\begin{array}{l}\text { Online } \\
\text { survey; } 2010\end{array}$ & $\begin{array}{l}\text { The Netherlands; quota } \\
\text { sample, } N=1083,50 \% \text { men }\end{array}$ & $\begin{array}{l}\text { Motivational } \\
\text { explanations for } \\
\text { responses to the } \\
\text { meat-free meal } \\
\text { idea }\end{array}$ & $\begin{array}{l}\text { No info before } \\
\text { questions }\end{array}$ & $\begin{array}{l}\text { (1) Agriculture and } \\
\text { animal husbandry } \\
\text { together are one of the } \\
\text { major causes of climate } \\
\text { change. } \\
\text { (2) If agriculture and } \\
\text { animal husbandry change } \\
\text { the way they work, they } \\
\text { can counter climate } \\
\text { change. }\end{array}$ & $\begin{array}{l}\text { (1) } 23 \% \text { agree } \\
36 \% \text { unsure } \\
41 \% \text { disagree } \\
\text { (2) } 38 \% \text { agree } \\
37 \% \text { unsure } \\
25 \% \text { disagree }\end{array}$ & n.a. \\
\hline
\end{tabular}


Table A1. Cont.

\begin{tabular}{|c|c|c|c|c|c|c|c|}
\hline \multicolumn{5}{|l|}{ Title } & \multicolumn{3}{|c|}{ Outcome Measure: Perceived Environmental Impact } \\
\hline $\begin{array}{l}\text { Author(s), } \\
\text { Year }\end{array}$ & $\begin{array}{l}\text { Design; Year } \\
\text { Data Collected }\end{array}$ & Country; Sample & $\begin{array}{l}\text { Main Research } \\
\text { Question }\end{array}$ & $\begin{array}{l}\text { Provided } \\
\text { Information Prior } \\
\text { to the Experiment }\end{array}$ & $\begin{array}{l}\text { Question or Dependent } \\
\text { Variable }\end{array}$ & Response or Finding & Effect of Covariates \\
\hline $\begin{array}{l}\text { Study } 2 \\
\text { de Boer et al., } \\
2014 \text { [31] * }\end{array}$ & $\begin{array}{l}\text { Online } \\
\text { survey; } 2010\end{array}$ & $\begin{array}{l}\text { The Netherlands; } \\
\text { quota sample, } N=1083, \\
50 \% \text { men }\end{array}$ & $\begin{array}{l}\text { Consumer } \\
\text { strategies to } \\
\text { reduce meat } \\
\text { consumption and } \\
\text { its' association } \\
\text { with their } \\
\text { willingness to eat } \\
\text { meatless meals }\end{array}$ & $\begin{array}{l}\text { As an individual, } \\
\text { you can make a } \\
\text { big difference to } \\
\text { nature and } \\
\text { climate protection } \\
\text { by choosing one } \\
\text { (or more) meals } \\
\text { without meat } \\
\text { every week. }\end{array}$ & Did you know that? & $64 \%$ yes, $36 \%$ no & $\begin{array}{l}\text { More 'yes' responses for } \\
\text { older and better educated } \\
\text { people }\end{array}$ \\
\hline $\begin{array}{l}\text { De Groeve, } \\
\text { et al., } 2017 \text { [21] }\end{array}$ & $\begin{array}{l}\text { Online survey. } \\
\text { Two samples. } \\
\text { Data collected } \\
\text { in } 2015 \\
\text { (sample 1) and } \\
2016 \text { (sample 2) }\end{array}$ & $\begin{array}{l}\text { Belgium; Ghent University } \\
\text { Business Administration } \\
\text { Students; } N=429\end{array}$ & $\begin{array}{l}\text { Assess students } \\
\text { support for six less } \\
\text { meat initiatives } \\
\text { (LMIs) to be } \\
\text { implemented in } \\
\text { student } \\
\text { restaurants. }\end{array}$ & $\begin{array}{l}\text { No prior info } \\
\text { given. }\end{array}$ & $\begin{array}{l}\text { Students' knowledge } \\
\text { about the negative impact } \\
\text { of meat on the } \\
\text { environment }\end{array}$ & $\begin{array}{l}4.66 \% \text { reported "Very much" } \\
24.4 \% \text { rather much } \\
36.6 \% \text { not little, not much } \\
24.4 \% \text { Little } \\
9.79 \% \text { Very little }\end{array}$ & n.a. \\
\hline $\begin{array}{l}\text { Graca, } \\
\text { Oliveira, et al., } \\
2015 \text { [24] * }\end{array}$ & $\begin{array}{l}\text { Online } \\
\text { survey; } 2013\end{array}$ & $\begin{array}{l}\text { Portugal; convenience } \\
\text { sample, } N=410,30 \% \text { men }\end{array}$ & $\begin{array}{l}\text { Multiple } \\
\text { correspondence } \\
\text { analysis to } \\
\text { identify clusters } \\
\text { of meat-related } \\
\text { associations }\end{array}$ & $\begin{array}{l}\text { Info provided } \\
\text { after the question }\end{array}$ & $\begin{array}{l}\text { Participants responded to } \\
\text { an open ended question } \\
\text { about how meat } \\
\text { consumption may impact } \\
\text { nature and the } \\
\text { environment }\end{array}$ & $\begin{array}{l}24 \% \text { pollutes nature and the } \\
\text { environment; } 20 \% \text { erosion, } \\
\text { disruption, depletion of } \\
\text { natural resources; } 18 \% \\
\text { references to mass } \\
\text { production, artificial } \\
\text { methods; } 14 \% \text { impacts only } \\
\text { if unregulated or in excess; } \\
11 \% \text { does not impact nature } \\
\text { and the environment; }\end{array}$ & n.a. \\
\hline $\begin{array}{l}\text { Lea \& } \\
\text { Worsley, } \\
2008[27]^{*}\end{array}$ & $\begin{array}{l}\text { Postal survey; } \\
2004\end{array}$ & $\begin{array}{l}\text { Australia (Victoria); } \\
\text { random sample, } \\
N=223,48 \% \text { men }\end{array}$ & $\begin{array}{l}\text { Food-related } \\
\text { environmental } \\
\text { beliefs and } \\
\text { behaviors }\end{array}$ & No info & $\begin{array}{l}\text { Consumers eating less } \\
\text { meat' is important to help } \\
\text { the environment }\end{array}$ & $\begin{array}{l}22 \% \text { agree } \\
22 \% \text { unsure } \\
56 \% \text { disagree } \\
\text { Lowest level of agreement } \\
\text { compared with other } \\
\text { behaviors } \\
\text { (e.g., using less packaging, } \\
\text { grown locally) }\end{array}$ & n.a. \\
\hline
\end{tabular}


Table A1. Cont.

\begin{tabular}{|c|c|c|c|c|c|c|c|}
\hline \multicolumn{5}{|l|}{ Title } & \multicolumn{3}{|c|}{ Outcome Measure: Perceived Environmental Impact } \\
\hline $\begin{array}{l}\text { Author(s), } \\
\text { Year }\end{array}$ & $\begin{array}{l}\text { Design; Year } \\
\text { Data Collected }\end{array}$ & Country; Sample & $\begin{array}{l}\text { Main Research } \\
\text { Question }\end{array}$ & $\begin{array}{l}\text { Provided } \\
\text { Information Prior } \\
\text { to the Experiment }\end{array}$ & $\begin{array}{l}\text { Question or Dependent } \\
\text { Variable }\end{array}$ & Response or Finding & Effect of Covariates \\
\hline $\begin{array}{l}\text { Pohjolainen } \\
\text { et al., 2016 [22] }\end{array}$ & $\begin{array}{l}\text { Postal survey; } \\
2010\end{array}$ & $\begin{array}{l}\text { Finland; } \\
\text { representative sample. } \\
N=1890\end{array}$ & $\begin{array}{l}\text { The level of } \\
\text { environmental } \\
\text { consciousness } \\
\text { among } \\
\text { Finnish consumers } \\
\text { concerning meat } \\
\text { production and } \\
\text { consumption }\end{array}$ & $\begin{array}{l}\text { No prior info } \\
\text { given. }\end{array}$ & $\begin{array}{l}\text { Participants had to agree } \\
\text { or disagree with the } \\
\text { following three } \\
\text { statements: } \\
\text { (1) meat production } \\
\text { strengthens climate } \\
\text { change significantly more } \\
\text { than plant production } \\
\text { (2) meat production } \\
\text { causes eutrophication } \\
\text { significantly more than } \\
\text { plant production } \\
\text { (3) food production causes } \\
\text { significant environmental } \\
\text { problems }\end{array}$ & $\begin{array}{l}\text { (1) } 35.7 \% \text { agree; } 47 \% \text { neutral; } \\
17.3 \% \text { disagree } \\
\text { (2) } 34.8 \% \text { agree; } 45 \% \\
\text { neutral; } 20.2 \% \text { disagree } \\
\text { (3) } 35.6 \% \text { agree; } 37.7 \% \\
\text { neutral; } 26.7 \% \text { disagree }\end{array}$ & $\begin{array}{l}\text { Consumers clustered in six } \\
\text { groups depending on their } \\
\text { awareness of meat-related } \\
\text { environmental questions: } \\
\text { Those aware (highly } \\
\text { conscious and rather } \\
\text { conscious), those resistant } \\
\text { to the idea (Resistant), those } \\
\text { who give neutral answers } \\
\text { (highly unsure and rather } \\
\text { unsure) and those "careless } \\
\text { conscious". } \\
\text { Among the groups highly } \\
\text { and rather conscious, the } \\
\text { majority is female (66.2\% } \\
\text { and } 55.3 \% \text { ), two thirds aged } \\
\text { between } 46-75,40 \% \text { or more } \\
\text { have tertiary education. } \\
\text { When occupation is } \\
\text { considered, in both groups } \\
\text { more than } 40 \% \text { are not in } \\
\text { labor force and blue-collar } \\
\text { workers are slightly more } \\
\text { represented than } \\
\text { white-collar } \\
\text { (26.1-21.1\%/21.9-19.6\%). }\end{array}$ \\
\hline
\end{tabular}


Table A1. Cont.

\begin{tabular}{|c|c|c|c|c|c|c|c|}
\hline \multicolumn{5}{|l|}{ Title } & \multicolumn{3}{|c|}{ Outcome Measure: Perceived Environmental Impact } \\
\hline $\begin{array}{l}\text { Author(s), } \\
\text { Year }\end{array}$ & $\begin{array}{l}\text { Design; Year } \\
\text { Data Collected }\end{array}$ & Country; Sample & $\begin{array}{l}\text { Main Research } \\
\text { Question }\end{array}$ & $\begin{array}{l}\text { Provided } \\
\text { Information Prior } \\
\text { to the Experiment }\end{array}$ & $\begin{array}{l}\text { Question or Dependent } \\
\text { Variable }\end{array}$ & Response or Finding & Effect of Covariates \\
\hline $\begin{array}{l}\text { Tobler et al., } \\
2011 \text { [33] } \\
\text { follow-up } \\
\text { study by } \\
\text { Siegrist et al., } \\
2015 \text { [32] * }\end{array}$ & $\begin{array}{l}\text { Postal survey; } \\
\text { longitudinal } \\
\text { study: 2010, } \\
\text { follow-up } \\
2014\end{array}$ & $\begin{array}{l}\text { Switzerland } \\
\text { (German- and } \\
\text { French-speaking } \\
\text { regions); } \\
\text { random panel } \\
\text { sample, } \\
\text { N2010 = } 6189, \\
\text { N2014 = 2781, } \\
48 \% \text { men }\end{array}$ & $\begin{array}{l}\text { Consumer } \\
\text { willingness to } \\
\text { adopt ecological } \\
\text { food consumption }\end{array}$ & No info & $\begin{array}{l}\text { Perceived environmental } \\
\text { benefit of eating less meat } \\
\text { (maximum of once } \\
\text { or twice per week), } \\
\text { ( } 1 \text { = very small to } 6 \text { = very } \\
\text { large) }\end{array}$ & $\begin{array}{l}\mathrm{M}=3.75, \mathrm{SD}=1.71 \text {, } \\
\text { reducing meat consumption } \\
\text { was perceived as having the } \\
\text { lowest environmental effect } \\
\text { compared with other } \\
\text { behaviors (e.g., avoiding } \\
\text { excessive packaging or } \\
\text { organic food). } \\
\\
\text { Longitudinal study; } \\
\text { Increase across time } \\
(\mathrm{M} 2010=3.89, \\
\mathrm{SD}=1.69 ; \mathrm{M} 2014=4.23, \\
\mathrm{SD}=1.56 ; p<0.001)\end{array}$ & $\begin{array}{l}\text { Women perceived meat } \\
\text { reduction as more beneficial } \\
\text { for the environment than } \\
\text { men } \\
(\mathrm{M}=3.96, \mathrm{SD}=1.69 ; \\
\mathrm{M}=3.52, \mathrm{SD}=1.70 ; \\
p<0.001) \\
\text { Larger improvement for } \\
\text { women and higher } \\
\text { educated participants; } \\
p<0.001\end{array}$ \\
\hline
\end{tabular}


Table A1. Cont.

\begin{tabular}{|c|c|c|c|c|c|c|c|}
\hline \multicolumn{5}{|l|}{ Title } & \multicolumn{3}{|c|}{ Outcome Measure: Perceived Environmental Impact } \\
\hline $\begin{array}{l}\text { Author(s), } \\
\text { Year }\end{array}$ & $\begin{array}{l}\text { Design; Year } \\
\text { Data Collected }\end{array}$ & Country; Sample & $\begin{array}{l}\text { Main Research } \\
\text { Question }\end{array}$ & $\begin{array}{l}\text { Provided } \\
\text { Information Prior } \\
\text { to the Experiment }\end{array}$ & $\begin{array}{c}\text { Question or Dependent } \\
\text { Variable }\end{array}$ & Response or Finding & Effect of Covariates \\
\hline $\begin{array}{l}\text { Truelove et al., } \\
2012 \text { [26] }\end{array}$ & $\begin{array}{l}\text { Mixed methods. } \\
\text { Online survey } \\
\text { with open } \\
\text { ended } \\
\text { questions and } \\
\text { behavior } \\
\text { ratings.; } 2008\end{array}$ & $\begin{array}{l}\text { USA; Undergraduate } \\
\text { psychology majors }(N=112) \\
(69 \text { women and } 43 \text { men })\end{array}$ & $\begin{array}{l}\text { Students } \\
\text { perceptions of the } \\
\text { relative impact } \\
\text { and effectiveness } \\
\text { of certain } \\
\text { behaviors on } \\
\text { global warming. }\end{array}$ & $\begin{array}{l}\text { No prior info } \\
\text { given. }\end{array}$ & $\begin{array}{l}\text { (1) Open-ended request to } \\
\text { participants to list their } \\
\text { own behaviors that cause } \\
\text { global warming. } \\
\text { (2) Respondents asked to } \\
\text { rate the impact of } 16 \\
\text { behaviors in contributing } \\
\text { to GW. Rate went from } 1 \\
\text { (Negligible impact) to } 11 \\
\text { (Major impact). } \\
\text { (3) Open-ended request to } \\
\text { participants to list } \\
\text { behaviors that reduce } \\
\text { global warming. } \\
\text { (4) Respondents asked to } \\
\text { rate the impact of } 20 \\
\text { behaviors that contribute } \\
\text { to reduce GW. } 1 \\
\text { (Extremely ineffective) to } \\
11 \text { (Extremely effective) }\end{array}$ & $\begin{array}{l}\text { (1) Driving was mentioned } \\
\text { by } 90 \% \text { participants. Eat } \\
\text { meat only by less than } 10 \% \\
\text { (2) Eat meat was rated with } \\
\text { median of } 3.83 / 11 \text {, just } \\
\text { above behaviors like riding } \\
\text { your bike and skiing. SD: } \\
2.52 \\
\text { (3) Drive less and use } \\
\text { alternate transportation was } \\
\text { mentioned by almost } 80 \% \\
\text { of the participants. Recycle } \\
\text { by more than } 45 \% \text {. Reduce } \\
\text { meat consumption by less } \\
\text { than } 5 \% \\
\text { (4) Reduce your meat } \\
\text { consumption: } 4.35 / 11 \\
\text { effectiveness. SD: } 2.96\end{array}$ & $\begin{array}{l}\text { In answer (4), women } \\
\text { scored higher than men. }\end{array}$ \\
\hline $\begin{array}{l}\text { Vanhonacker } \\
\text { et al., } 2013 \\
{[23]^{*}}\end{array}$ & $\begin{array}{l}\text { Online } \\
\text { survey; } 2011\end{array}$ & $\begin{array}{l}\text { Belgium } \\
\text { (Flanders); } \\
\text { convenience } \\
\text { sample, } \\
N=221,36 \% \\
\text { men }\end{array}$ & $\begin{array}{l}\text { Attitudes } \\
\text { towards more } \\
\text { sustainable } \\
\text { food choices } \\
\text { and consumer } \\
\text { segmentation } \\
\text { based on their } \\
\text { self-evaluated } \\
\text { ecological } \\
\text { footprint }\end{array}$ & $\begin{array}{l}\text { Explanation of } \\
\text { the concept } \\
\text { 'ecological } \\
\text { footprint' } \\
\text { Participants were } \\
\text { informed about } \\
\text { the contribution } \\
\text { of animal } \\
\text { production to Co2 } \\
\text { emissions. }\end{array}$ & $\begin{array}{l}\text { Participants had to score } \\
\text { the contribution to } \\
\text { greenhouse gas emissions } \\
\text { for various industry } \\
\text { sectors, including } \\
\text { livestock production. } \\
\text { Participants were asked } \\
\text { how aware they were of } \\
\text { the extent of this } \\
\text { contribution. }\end{array}$ & $\begin{array}{l}\text { Approx. } \mathrm{M}=3.7 \text { (no } \\
\text { number, only bar chart } \\
\text { presented) } \\
\text { (1= does not contribute at } \\
\text { all to } 5=\text { contributes very } \\
\text { much) } \\
\text { Livestock production was } \\
\text { underestimated relative to } \\
\text { other activities (e.g., } \\
\text { transport, energy use) } \\
58 \% \text { reported awareness }\end{array}$ & n.a. \\
\hline
\end{tabular}

Notes n.a.: not assessed; $\mathrm{M}$ = arithmetic mean; $\mathrm{SD}$ = standard deviation; $\mathrm{SES}=$ socioeconomic status. *: As reported by [55]. 


\section{Appendix B}

Table A2. People willingness to stop or reduce meat consumption because of its environmental impact.

\begin{tabular}{|c|c|c|c|c|c|c|c|}
\hline \multirow[b]{2}{*}{$\begin{array}{l}\text { Author(s), } \\
\text { Year }\end{array}$} & \multirow[b]{2}{*}{$\begin{array}{l}\text { Design; Year } \\
\text { Data Collected }\end{array}$} & \multirow[b]{2}{*}{ Country; Sample } & \multirow[b]{2}{*}{$\begin{array}{l}\text { Main Research } \\
\text { Question }\end{array}$} & \multirow[b]{2}{*}{$\begin{array}{l}\text { Provided } \\
\text { Information Prior } \\
\text { the Experiment }\end{array}$} & \multicolumn{3}{|c|}{ Outcome Measure: Willingness to Reduce/Replace } \\
\hline & & & & & $\begin{array}{l}\text { Question or Dependent } \\
\text { Variable }\end{array}$ & Response or Finding & Effect of Covariates \\
\hline $\begin{array}{l}\text { Campbell- } \\
\text { Arvai et al., } \\
2014[29]^{*}\end{array}$ & $\begin{array}{l}\text { Experimental } \\
\text { between- } \\
\text { subject design } \\
\text { with control } \\
\text { group; } \\
\text { unspecified. }\end{array}$ & $\begin{array}{l}\text { U.S.; convenience sample of } \\
\text { students, } N=319,46 \% \text { men }\end{array}$ & $\begin{array}{l}\text { Nudging } \\
\text { intervention; } \\
\text { food-related } \\
\text { environmental } \\
\text { beliefs and } \\
\text { behaviors }\end{array}$ & $\begin{array}{l}\text { Use of a default } \\
\text { vegetarian meal } \\
\text { option vs. } \\
\text { provision of } \\
\text { information on the } \\
\text { menus. }\end{array}$ & $\begin{array}{l}\text { Hypothetical choice of a } \\
\text { lunch or dinner meal } \\
\text { (with or without meat) }\end{array}$ & $\begin{array}{l}\text { Offering a vegetarian } \\
\text { option as default increased } \\
\text { the probability that } \\
\text { participants would choose a } \\
\text { meat-free meal (OR }=4.10, \\
p<0.001) \text {, information on } \\
\text { the menu did not } \\
\text { significantly influence meal } \\
\text { choice }(\mathrm{OR}=1.09, p=534) .\end{array}$ & $\begin{array}{l}\text { Females were more likely tc } \\
\text { choose meat-free menus } \\
(\mathrm{OR}=0.49, p=0.02), \\
\text { biospheric value orientation } \\
\text { and pro-environmental } \\
\text { beliefs were not significant }\end{array}$ \\
\hline $\begin{array}{l}\text { Cordts et al., } \\
2014[25]^{*}\end{array}$ & $\begin{array}{l}\text { Online } \\
\text { experiment; } \\
2013\end{array}$ & $\begin{array}{l}\text { Germany; quota sample, } \\
N=590,52 \% \text { men }\end{array}$ & $\begin{array}{l}\text { Consumer } \\
\text { response to } \\
\text { negative } \\
\text { information on } \\
\text { meat consumption }\end{array}$ & $\begin{array}{l}\text { Randomization to } \\
\text { info about } \\
\text { negative } \\
\text { consequences of } \\
\text { meat consumption } \\
\text { for animal } \\
\text { welfare/health/climat } \\
\text { change/personal } \\
\text { image; no control } \\
\text { group. }\end{array}$ & $\begin{array}{l}\text { Consumers' belief that } \\
\text { they will reduce their } \\
\text { meat consumption in the } \\
\text { future (measured before } \\
\text { and after info) } \\
\text { ate }\end{array}$ & $\begin{array}{l}\text { Before info: } 12.8 \% \\
\text { After info: } 18.8 \% \text { (climate } \\
\text { change) to } 28.0 \% \text { (animal } \\
\text { welfare) }\end{array}$ & $\begin{array}{l}\text { Condition climate change: } \\
\text { Smaller effect in men } \\
\text { compared with women } \\
(15.5 \% \text { vs. } 22.8 \%)\end{array}$ \\
\hline
\end{tabular}


Table A2. Cont.

\begin{tabular}{|c|c|c|c|c|c|c|c|}
\hline \multirow[b]{2}{*}{$\begin{array}{l}\text { Author(s), } \\
\text { Year }\end{array}$} & \multirow[b]{2}{*}{$\begin{array}{l}\text { Design; Year } \\
\text { Data Collected }\end{array}$} & \multirow[b]{2}{*}{ Country; Sample } & \multirow[b]{2}{*}{$\begin{array}{l}\text { Main Research } \\
\text { Question }\end{array}$} & \multirow[b]{2}{*}{$\begin{array}{l}\text { Provided } \\
\text { Information Prior } \\
\text { the Experiment }\end{array}$} & \multicolumn{3}{|c|}{ Outcome Measure: Willingness to Reduce/Replace } \\
\hline & & & & & $\begin{array}{c}\text { Question or Dependent } \\
\text { Variable }\end{array}$ & Response or Finding & Effect of Covariates \\
\hline $\begin{array}{l}\text { De Boer et al., } \\
2018[38]\end{array}$ & $\begin{array}{l}\text { Survey data } \\
\text { obtained from } \\
\text { EU Report; } 2012\end{array}$ & See EU Report & See EU Report & See EU Report & See EU Report & See EU Report. & $\begin{array}{l}\text { (1) Willingness to replace } \\
\text { meat (\%yes) } \\
\text { (2) Willingness to eat less } \\
\text { but better meat (\%yes) } \\
\text { High-income zones } \\
\text { Northern zone (1) } 38 \% \\
\text { (2) } 77 \% \\
\text { Western Central zone } \\
\text { (1) } 42 \% \text { (2) } 78 \% \\
\text { Medit. zone (1) } 55 \% \text { (2) } 86 \% \\
\text { Medium-to-low income } \\
\text { zones } \\
\text { Northern zone (1) } 54 \% \\
\text { (2) } 83 \% \\
\text { Western Central zone } \\
\text { (1) } 63 \% \text { (2) } 86 \% \\
\text { Medit. zone (1) } 57 \% \text { (2) } 86 \%\end{array}$ \\
\hline $\begin{array}{l}\text { Study 1: } \\
\text { de Boer, } \\
\text { Schösler, et al., } \\
2013 \text { [20] * }\end{array}$ & $\begin{array}{l}\text { Online survey; } \\
2010\end{array}$ & $\begin{array}{l}\text { Netherlands; } \\
\text { quota sample, } N=1083 \text {, } \\
50 \% \text { men }\end{array}$ & $\begin{array}{l}\text { Motivational } \\
\text { explanations for } \\
\text { responses to the } \\
\text { meat-free meal } \\
\text { idea }\end{array}$ & $\begin{array}{l}\text { As an individual, } \\
\text { you can make a } \\
\text { big difference to } \\
\text { nature and climate } \\
\text { protection by } \\
\text { choosing one (or } \\
\text { more) meals } \\
\text { without meat } \\
\text { every week. }\end{array}$ & $\begin{array}{l}\text { Willingness to choose one } \\
\text { or more meals without } \\
\text { meat every week }\end{array}$ & $\begin{array}{l}5 \% \text { certainly } \\
41 \% \text { maybe } \\
21 \% \text { doing so already } \\
23 \% \text { do not want to }\end{array}$ & $\begin{array}{l}\text { Predictors for 'does not } \\
\text { want to change' vs. 'maybe' } \\
\text { (reference): skepticism } \\
\text { about climate change } \\
(\mathrm{OR}=1.98, p<0.001), \text { value } \\
\text { of care for nature (OR }=0.64, \\
p<0.001) \text {, level of education } \\
(\mathrm{OR}=0.90, p<0.05) \text { (based } \\
\text { on standardized predictors) }\end{array}$ \\
\hline
\end{tabular}


Table A2. Cont.

\begin{tabular}{|c|c|c|c|c|c|c|c|}
\hline \multirow[b]{2}{*}{$\begin{array}{l}\text { Author(s), } \\
\text { Year }\end{array}$} & \multirow[b]{2}{*}{$\begin{array}{l}\text { Design; Year } \\
\text { Data Collected }\end{array}$} & \multirow[b]{2}{*}{ Country; Sample } & \multirow[b]{2}{*}{$\begin{array}{l}\text { Main Research } \\
\text { Question }\end{array}$} & \multirow[b]{2}{*}{$\begin{array}{l}\text { Provided } \\
\text { Information Prior } \\
\text { the Experiment }\end{array}$} & \multicolumn{3}{|c|}{ Outcome Measure: Willingness to Reduce/Replace } \\
\hline & & & & & $\begin{array}{l}\text { Question or Dependent } \\
\text { Variable }\end{array}$ & Response or Finding & Effect of Covariates \\
\hline $\begin{array}{l}\text { Study 2: } \\
\text { de Boer et al., } \\
2014 \text { [31] }\end{array}$ & [the same] & [the same] & $\begin{array}{l}\text { Consumers' } \\
\text { strategies to } \\
\text { reduce meat } \\
\text { consumption and } \\
\text { its' association } \\
\text { with their } \\
\text { willingness to eat } \\
\text { meat-less meals. }\end{array}$ & [the same] & $\begin{array}{l}\text { Willingness to choose one } \\
\text { or more meals without } \\
\text { meat every week }\end{array}$ & Same results as in study 1 & $\begin{array}{l}\text { Predictors for 'certainly' vs. } \\
\text { 'maybe' (reference): Female } \\
\text { gender }(\mathrm{OR}=2.02, p<0.01) \text {, } \\
\text { familiarity with topic } \\
(\mathrm{OR}=2.67, p<0.001), \\
\text { buying meat substitutes } \\
(\mathrm{OR}=1.39, p<0.001) \text {, } \\
\text { preference for plant-based } \\
\text { proteins }(\mathrm{OR}=1.34, p<0.01) \\
\text { and number of meat-eating } \\
\text { days }(\mathrm{OR}=0.70, p<0.001) ; \\
\text { education and age were n.s. }\end{array}$ \\
\hline $\begin{array}{l}\text { De Boer et al., } \\
2016 \text { [30] }\end{array}$ & $\begin{array}{l}\text { Nation-wide } \\
\text { consumer } \\
\text { surveys; } 2014\end{array}$ & $\begin{array}{l}\text { Netherlands and the USA; } \\
\text { representative sample } \\
N=527 \text { (Netherlands) } \\
\text { Weighted variables: gender, } \\
\text { age, level of education, } \\
\text { region, and a value-related } \\
\text { test score on } \\
\text { "mentality-environment". } \\
\text { (efficiency of the weighting } \\
89 \% \text {, effective sample size } \\
478 \text { ) } \\
N=556 \text { (USA). } \\
\text { Weighted variables: gender, } \\
\text { age, and level of education } \\
\text { (efficiency of the weighting } \\
90 \% \text {, effective sample size } \\
500) \text { Total = } 1083\end{array}$ & $\begin{array}{l}\text { Consumers } \\
\text { awareness of meat } \\
\text { consumption } \\
\text { environmental } \\
\text { impact and their } \\
\text { willingness to } \\
\text { reduce meat } \\
\text { consumption, } \\
\text { among other } \\
\text { research questions. }\end{array}$ & $\begin{array}{l}\text { No prior info } \\
\text { given. }\end{array}$ & $\begin{array}{l}\text { Willingness to personally } \\
\text { make lifestyle-changes } \\
\text { (those already doing it at } \\
\text { the time of experiment } \\
\text { were instructed to choose } \\
\text { the option "certainly } \\
\text { willing")." The answer } \\
\text { categories were "Certainly } \\
\text { not willing" (1), "Likely } \\
\text { not willing" (2), "Likely } \\
\text { willing" (4), "Certainly } \\
\text { willing" (5), and "Don't } \\
\text { know" (recoded to 3). }\end{array}$ & $\begin{array}{l}\text { Only a small group of } \\
\text { participants of both } \\
\text { countries were willing to } \\
\text { change. Reducing meat } \\
\text { consumption was the } \\
\text { second less chosen behavior } \\
\text { to curb climate change } \\
\text { among the DUTCH } \\
(\mathrm{M}=3.58 \mathrm{SD}=0.36) \text { and the } \\
\text { least chosen among the U.S. } \\
(\mathrm{M}=3.01 \mathrm{SD}=1.44)\end{array}$ & $\begin{array}{l}\text { When participants believed } \\
\text { eating less meat to be a } \\
\text { highly effective behavior to } \\
\text { curb climate change, the } \\
\text { medians increased: Dutch } \\
\mathrm{M}=4.26 \mathrm{SD}=0.96 ; \text { US } \\
\mathrm{M}=3.88 \mathrm{SD}=1.19 \text {. }\end{array}$ \\
\hline
\end{tabular}


Table A2. Cont.

\begin{tabular}{|c|c|c|c|c|c|c|c|}
\hline \multirow[b]{2}{*}{$\begin{array}{l}\text { Author(s), } \\
\text { Year }\end{array}$} & \multirow[b]{2}{*}{$\begin{array}{l}\text { Design; Year } \\
\text { Data Collected }\end{array}$} & \multirow[b]{2}{*}{ Country; Sample } & \multirow[b]{2}{*}{$\begin{array}{l}\text { Main Research } \\
\text { Question }\end{array}$} & \multirow[b]{2}{*}{$\begin{array}{l}\text { Provided } \\
\text { Information Prior } \\
\text { the Experiment }\end{array}$} & \multicolumn{3}{|c|}{ Outcome Measure: Willingness to Reduce/Replace } \\
\hline & & & & & $\begin{array}{l}\text { Question or Dependent } \\
\text { Variable }\end{array}$ & Response or Finding & Effect of Covariates \\
\hline $\begin{array}{l}\text { De Groeve, } \\
\text { et al., 2017 [21] }\end{array}$ & $\begin{array}{l}\text { Online survey. } \\
\text { Two samples. } \\
\text { Data collected } \\
\text { in } 2015 \text { (sample } \\
\text { 1) and } 2016 \\
\text { (sample 2) }\end{array}$ & $\begin{array}{l}\text { Belgium; } \\
\text { Ghent University Business } \\
\text { Administration Students } \\
N=429\end{array}$ & $\begin{array}{l}\text { Assess students } \\
\text { support for six less } \\
\text { meat initiatives } \\
\text { (LMIs) to be } \\
\text { implemented in } \\
\text { student } \\
\text { restaurants. }\end{array}$ & $\begin{array}{l}\text { Each respondent } \\
\text { had a } 50 \% \text { chance } \\
\text { of receiving } \\
\text { information about } \\
\text { the climate impact } \\
\text { of meat before } \\
\text { assessing their } \\
\text { support for the } \\
\text { LMIs }\end{array}$ & $\begin{array}{l}\text { Support for indirect and } \\
\text { direct meat curtailment } \\
\text { actions: } \\
\text { DIRECT MEAT } \\
\text { CURTAILMENT } \\
\text { "Eating beef or mutton } \\
\text { once a week at } \\
\text { maximum." M } \\
\text { "Reduce your portions of } \\
\text { meat per meal (for } \\
\text { example, } 100 \text { g instead of } \\
120 \mathrm{~g}) \mathrm{P} \\
\text { "Increase the supply of } \\
\text { vegetarian main meals up } \\
\text { to } 50 \% \text { of the meals." V } \\
\\
\text { "Switching to a 'contrarian } \\
\text { week' in student } \\
\text { restaurants whereby } \\
\text { meals with meat are } \\
\text { served one day a week, } \\
\text { and vegetarian meals four } \\
\text { days a week." C }\end{array}$ & $\begin{array}{l}\text { DIRECT MEAT } \\
\text { CURTAILMENT } \\
\text { STRATEGIES: } \\
\text { M } \\
\text { Strongly disagree 20\% } \\
\text { Tend to disagree } 27 \% \\
\text { Neutral } 21 \% \\
\text { Tend to agree } 25 \% \\
\text { Strongly agree } 9 \% \\
\text { P } \\
\text { Strongly disagree } 9 \% \\
\text { Tend to disagree } 15 \% \\
\text { Neutral } 17 \% \\
\text { Tend to agree } 41 \% \\
\text { Strongly agree } 17 \% \\
\\
\text { V } \\
\text { Strongly disagree } 12 \% \\
\text { Tend to disagree } 26 \% \\
\text { Neutral } 28 \% \\
\text { Tend to agree } 24 \% \\
\text { Strongly agree } 10 \% \\
\\
\text { C } \\
\text { Strongly disagree } 35 \% \\
\text { Tend to disagree } 33 \% \\
\text { Neutral } 17 \% \\
\text { Tend to agree } 11 \% \\
\text { Strongly agree } 4 \%\end{array}$ & $\begin{array}{l}\text { A higher concern for } \\
\text { environmental problems is } \\
\text { correlated with more positive } \\
\text { appraisals of all the LMIs } \\
\text { (each } p<0.001 \text { ). A higher } \\
\text { KNIM [knowledge about the } \\
\text { negative impact of meat }{ }^{* *} \text { ] is } \\
\text { also significantly (but less } \\
\text { strongly) associated with } \\
\text { more positive appraisals of all } \\
\text { LMIs, except for LMI-M. } \\
\text { Higher appraisals of the } \\
\text { direct strategies for meat } \\
\text { curtailment (LMIs M, P, V and } \\
\text { C) are highly significantly } \\
\text { associated with sex and meat } \\
\text { consumption frequencies: } \\
\text { female students and students } \\
\text { who eat meat (or fish) with } \\
\text { their main meals less often } \\
\text { are more willing to support } \\
\text { these LMIs (in every case } \\
p<0.001 \text { ). Prior information } \\
\text { about the climate impact of } \\
\text { meat appears to have no } \\
\text { effect on the support for the } \\
\text { LMIs, except for LMI-C, } \\
\text { where there is a significant } \\
\text { negative effect of information } \\
\text { (U = 20,197; } p=0.024 \text { ) }\end{array}$ \\
\hline & & & & & & & $\begin{array}{l}* * \text { KNIM's four themes: } \\
\text { environment, animal welfare, } \\
\text { health, Global food } \\
\text { distribution. }\end{array}$ \\
\hline
\end{tabular}


Table A2. Cont.

\begin{tabular}{|c|c|c|c|c|c|c|c|}
\hline \multirow[b]{2}{*}{$\begin{array}{l}\text { Author(s), } \\
\text { Year }\end{array}$} & \multirow[b]{2}{*}{$\begin{array}{l}\text { Design; Year } \\
\text { Data Collected }\end{array}$} & \multirow[b]{2}{*}{ Country; Sample } & \multirow[b]{2}{*}{$\begin{array}{l}\text { Main Research } \\
\text { Question }\end{array}$} & \multirow[b]{2}{*}{$\begin{array}{l}\text { Provided } \\
\text { Information Prior } \\
\text { the Experiment }\end{array}$} & \multicolumn{3}{|c|}{ Outcome Measure: Willingness to Reduce/Replace } \\
\hline & & & & & $\begin{array}{l}\text { Question or Dependent } \\
\text { Variable }\end{array}$ & Response or Finding & Effect of Covariates \\
\hline $\begin{array}{l}\text { Graca, } \\
\text { Calheiros, } \\
\text { et al., 2015 } \\
{[37]^{*}}\end{array}$ & $\begin{array}{l}\text { Study 1: Online } \\
\text { survey; } 2014\end{array}$ & $\begin{array}{l}\text { Portugal; convenience } \\
\text { sample, } \\
N=1023,42 \% \text { men }\end{array}$ & $\begin{array}{l}\text { Development and } \\
\text { validation of a } \\
\text { meat attachment } \\
\text { questionnaire }\end{array}$ & $\begin{array}{l}\text { In recent times, } \\
\text { meat consumption } \\
\text { is being } \\
\text { increasingly } \\
\text { debated on the } \\
\text { grounds of } \\
\text { environmental } \\
\text { sustainability, } \\
\text { health and safety } \\
\text { concerns, and } \\
\text { animal } \\
\text { rights/welfare } \\
\text { arguments. }\end{array}$ & $\begin{array}{l}\text { Willingness to reduce } \\
\text { meat consumption } \\
\text { ( } 1=\text { not willing at all to } \\
5=\text { very willing). } \\
\text { Willingness to follow a } \\
\text { plant-based diet ( } 1=\text { not } \\
\text { willing at all to } 5=\text { very } \\
\text { willing) }\end{array}$ & No mean values presented. & $\begin{array}{l}\text { Predictors for meat } \\
\text { reduction: Meat attachment } \\
(\beta=-0.49, p<0.001) \text {, } \\
\text { positive attitudes towards } \\
\text { meat ( } \beta=-0.11, p<0.05) \\
\\
\text { Predictors for plant-based } \\
\text { diet: Meat attachment } \\
(\beta=-0.54, p<0.001) \text {, } \\
\text { positive attitudes towards } \\
\text { meat }(\beta=-0.12, p<0.05) \text {, } \\
\text { meat consumption } \\
\text { frequency }(\beta=-0.12, \\
p<0.01)\end{array}$ \\
\hline $\begin{array}{l}\text { Graca, } \\
\text { Calheiros, } \\
\text { et al., 2015 * }\end{array}$ & $\begin{array}{l}\text { Study 2: Online } \\
\text { survey; } 2015\end{array}$ & $\begin{array}{l}\text { Portugal; Amazon } \\
\text { Mechanical Turk, } N=318, \\
58 \% \text { men }\end{array}$ & $\begin{array}{l}\text { Predictive ability } \\
\text { of the meat } \\
\text { attachment } \\
\text { questionnaire for } \\
\text { willingness to } \\
\text { reduce meat } \\
\text { consumption. }\end{array}$ & see Study 1 & $\begin{array}{l}\text { Willingness and intention } \\
\text { to reduce meat } \\
\text { consumption, avoid } \\
\text { eating meat, follow a } \\
\text { plan-based diet (items } \\
\text { averaged for general } \\
\text { measure). }\end{array}$ & No mean values presented & $\begin{array}{l}\text { Predictors for willingness: } \\
\text { Meat attachment }(\beta=-0.75, \\
p<0.001), \text { PBC }(\beta=-0.12, \\
p<0.01) \\
\text { Predictors for intentions: } \\
\text { Attitudes towards meat } \\
(\beta=-0.32, p<0.001), \text { PBC } \\
(\beta=0.10, p<0.01), \text { meat } \\
\text { attachment ( } \beta=-0.53, \\
p<0.001) .\end{array}$ \\
\hline
\end{tabular}


Table A2. Cont.

\begin{tabular}{|c|c|c|c|c|c|c|c|}
\hline \multirow[b]{2}{*}{$\begin{array}{l}\text { Author(s), } \\
\text { Year }\end{array}$} & \multirow[b]{2}{*}{$\begin{array}{l}\text { Design; Year } \\
\text { Data Collected }\end{array}$} & \multirow[b]{2}{*}{ Country; Sample } & \multirow[b]{2}{*}{$\begin{array}{l}\text { Main Research } \\
\text { Question }\end{array}$} & \multirow[b]{2}{*}{$\begin{array}{l}\text { Provided } \\
\text { Information Prior } \\
\text { the Experiment }\end{array}$} & \multicolumn{3}{|c|}{ Outcome Measure: Willingness to Reduce/Replace } \\
\hline & & & & & $\begin{array}{l}\text { Question or Dependent } \\
\text { Variable }\end{array}$ & Response or Finding & Effect of Covariates \\
\hline $\begin{array}{l}\text { Graca, } \\
\text { Oliveira, et al., } \\
2015 \text { [24] }\end{array}$ & $\begin{array}{l}\text { Online } \\
\text { survey; } 2013\end{array}$ & $\begin{array}{l}\text { Portugal; } \\
\text { convenience } \\
\text { sample, } N=410,30 \% \text { men }\end{array}$ & $\begin{array}{l}\text { Multiple } \\
\text { correspondence } \\
\text { analysis to } \\
\text { identify clusters } \\
\text { of meat-related } \\
\text { associations }\end{array}$ & $\begin{array}{l}\text { Info was provided } \\
\text { related to the } \\
\text { negative } \\
\text { consequences of } \\
\text { meat production } \\
\text { and consumption } \\
\text { for animals, nature } \\
\text { and the } \\
\text { environment as } \\
\text { well as public } \\
\text { health }\end{array}$ & $\begin{array}{l}\text { Intent to change current } \\
\text { level of meat consumption } \\
\text { Willingness to reduce } \\
\text { meat consumption by half } \\
\text { Willingness to follow a } \\
\text { plant-based diet }\end{array}$ & $\begin{array}{l}60 \% \text { yes, } 27 \% \text { no, }(12 \% \text { no } \\
\text { meat consumers) } \\
49 \% \text { yes, } 38 \% \text { no, }(12 \% \text { no } \\
\text { meat consumers) } \\
44 \% \text { yes, } 53 \% \text { no }\end{array}$ & n.a. \\
\hline $\begin{array}{l}\text { Hunter et al., } \\
2016[34]\end{array}$ & $\begin{array}{l}\text { Postal survey. } \\
\text { Date not } \\
\text { specified. }\end{array}$ & $\begin{array}{l}\text { Sweden; } \\
\text { stratified simple random } \\
\text { sample of single family } \\
\text { homes. } 55 \% \text { males. } 89.5 \% \text { of } \\
\text { the sample had at least one } \\
\text { child. Mean age } 55 . \\
219 \text { usable questionnaires } \\
\text { were returned by post for a } \\
\text { response rate of } 22 \% \text { ( } 95 \% \\
\text { CI }(6.25)) \text {. }\end{array}$ & $\begin{array}{l}\text { Understand the } \\
\text { factors related to } \\
\text { fear or danger that } \\
\text { motivate } \\
\text { consumers to } \\
\text { reduce or alter } \\
\text { their meat } \\
\text { consumption. }\end{array}$ & $\begin{array}{l}\text { Yes, a cover story } \\
\text { stating the } \\
\text { negative impact of } \\
\text { climate change on } \\
\text { the earth and } \\
\text { humans and } \\
\text { statements about } \\
\text { the big impact } \\
\text { food has on } \\
\text { greenhouse gas } \\
\text { emissions as well } \\
\text { as statement that } \\
\text { reducing meat } \\
\text { consumption is the } \\
\text { most effective food } \\
\text { behavior that can } \\
\text { be adopted. }\end{array}$ & $\begin{array}{l}\text { Self-efficacy and response } \\
\text { efficacy questions } \\
\text { regarding meat } \\
\text { curtailment strategies }\end{array}$ & $\begin{array}{l}\text { At the same time, the mean } \\
\text { scores for self-efficacy and } \\
\text { response efficacy show that } \\
\text { the participants in this } \\
\text { study on average do not } \\
\text { find altered meat } \\
\text { consumption to be easy, nor } \\
\text { do they believe it to be very } \\
\text { effective. }\end{array}$ & \\
\hline
\end{tabular}


Table A2. Cont.

\begin{tabular}{|c|c|c|c|c|c|c|c|}
\hline \multirow[b]{2}{*}{$\begin{array}{l}\text { Author(s), } \\
\text { Year }\end{array}$} & \multirow[b]{2}{*}{$\begin{array}{l}\text { Design; Year } \\
\text { Data Collected }\end{array}$} & \multirow[b]{2}{*}{ Country; Sample } & \multirow[b]{2}{*}{$\begin{array}{l}\text { Main Research } \\
\text { Question }\end{array}$} & \multirow[b]{2}{*}{$\begin{array}{l}\text { Provided } \\
\text { Information Prior } \\
\text { the Experiment }\end{array}$} & \multicolumn{3}{|c|}{ Outcome Measure: Willingness to Reduce/Replace } \\
\hline & & & & & $\begin{array}{c}\text { Question or Dependent } \\
\text { Variable }\end{array}$ & Response or Finding & Effect of Covariates \\
\hline $\begin{array}{l}\text { Pohjolainen } \\
\text { et al., 2016 [22] }\end{array}$ & $\begin{array}{l}\text { Postal survey; } \\
2010\end{array}$ & $\begin{array}{l}\text { Finland; } \\
\text { representative sample. } \\
N=1890\end{array}$ & $\begin{array}{l}\text { The level of } \\
\text { environmental } \\
\text { consciousness } \\
\text { among } \\
\text { Finnish consumers } \\
\text { concerning meat } \\
\text { production and } \\
\text { consumption }\end{array}$ & $\begin{array}{l}\text { No prior info } \\
\text { given. }\end{array}$ & $\begin{array}{l}\text { Support to several actions } \\
\text { to curb the meat } \\
\text { production impact on the } \\
\text { environment }\end{array}$ & $\begin{array}{l}\text { Eating less meat the second } \\
\text { less supported, only after } \\
\text { techno-optimism; only } \\
25.5 \% \text { considered meat } \\
\text { reduction a possible } \\
\text { solution. } 39.2 \% \text { rejected this } \\
\text { choice. }\end{array}$ & $\begin{array}{l}\text { Consumers clustered in six } \\
\text { groups depending on their } \\
\text { awareness of meat-related } \\
\text { environmental questions: } \\
\text { Those aware (highly } \\
\text { conscious and rather } \\
\text { conscious), those resistant } \\
\text { to the idea (Resistant), those } \\
\text { who give neutral answers } \\
\text { (highly unsure and rather } \\
\text { unsure) and those "careless } \\
\text { conscious". } \\
\text { Among the highly } \\
\text { conscious, } 77.2 \% \text { agree with } \\
\text { meat reduction; among the } \\
\text { rather conscious, } 53 \% \text { agree } \\
\text { with meat reduction. }\end{array}$ \\
\hline $\begin{array}{l}\text { Schösler et al., } \\
2015 \text { [36] }^{*}\end{array}$ & $\begin{array}{l}\text { Face-to-face } \\
\text { interview; } 2013\end{array}$ & $\begin{array}{l}\text { Netherlands; quota samples } \\
\text { of second- generation } \\
\text { migrants: Turkish/Kurdish } \\
N=350 \text {, Chinese/Hong } \\
\text { Kongese } N=350 \text {, Native } \\
\text { Dutch } \\
N=357 ; 47-49 \% \text { men }\end{array}$ & $\begin{array}{l}\text { Gender differences } \\
\text { in meat } \\
\text { consumption and } \\
\text { reduction across } \\
\text { ethnic group }\end{array}$ & $\begin{array}{l}\text { As an individual, } \\
\text { you can make a } \\
\text { big difference to } \\
\text { nature and climate } \\
\text { protection by } \\
\text { choosing one (or } \\
\text { more) meals } \\
\text { without meat } \\
\text { every week. }\end{array}$ & $\begin{array}{l}\text { Willingness to reduce } \\
\text { meat consumption } \\
\text { (including 'yes', 'maybe') }\end{array}$ & $\begin{array}{l}\text { Willingness to reduce: } 17 \% \\
\text { Turks (monoculture), } 53 \% \\
\text { Chinese (monoculture), } 40 \% \\
\text { Native Dutch }\end{array}$ & $\begin{array}{l}\text { Turkish men followed by } \\
\text { Turkish women reported } \\
\text { lowest willingness to } \\
\text { reduce meat consumption; } \\
\text { no gender differences for } \\
\text { Native Dutch and Chinese. }\end{array}$ \\
\hline
\end{tabular}


Table A2. Cont.

\begin{tabular}{|c|c|c|c|c|c|c|c|}
\hline \multirow[b]{2}{*}{$\begin{array}{l}\text { Author(s), } \\
\text { Year }\end{array}$} & \multirow[b]{2}{*}{$\begin{array}{l}\text { Design; Year } \\
\text { Data Collected }\end{array}$} & \multirow[b]{2}{*}{ Country; Sample } & \multirow[b]{2}{*}{$\begin{array}{l}\text { Main Research } \\
\text { Question }\end{array}$} & \multirow[b]{2}{*}{$\begin{array}{l}\text { Provided } \\
\text { Information Prior } \\
\text { the Experiment }\end{array}$} & \multicolumn{3}{|c|}{ Outcome Measure: Willingness to Reduce/Replace } \\
\hline & & & & & $\begin{array}{l}\text { Question or Dependent } \\
\text { Variable }\end{array}$ & Response or Finding & Effect of Covariates \\
\hline $\begin{array}{l}\text { Tobler et al., } \\
2011[33]^{*}\end{array}$ & $\begin{array}{l}\text { Postal survey; } \\
2010\end{array}$ & $\begin{array}{l}\text { Switzerland (German- and } \\
\text { French-speaking regions); } \\
\text { random panel sample, } \\
40 \% \text { Native Dutch } \\
N=6189,48 \% \text { men }\end{array}$ & $\begin{array}{l}\text { Consumers' } \\
\text { willingness to } \\
\text { adopt ecological } \\
\text { food consumption }\end{array}$ & No info. & $\begin{array}{l}\text { Intention assessment } \\
\text { based on TTM for eating } \\
\text { less meat (maximum once } \\
\text { or twice per week) }\end{array}$ & $\begin{array}{l}\text { The largest fraction of } \\
\text { unwilling consumers was in } \\
\text { the domain of reducing } \\
\text { meat consumption. } \\
36.3 \% \text { (not willing) } 5.4 \% \\
\text { (willing but not ready) } 11.4 \\
\text { (willing and ready) } 46 \% \\
\text { (doing it already). Those in } \\
\text { the change stages (willing...) } \\
\text { were influenced by } \\
\text { environmental reasons. } \\
\text { Those doing it already were } \\
\text { influenced by health } \\
\text { reasons. }\end{array}$ & $\begin{array}{l}\text { Female gender }(\mathrm{OR}=1.76) \text {, } \\
\text { importance of naturalness } \\
(\mathrm{OR}=1.32) \text {, less meat is } \\
\text { healthier }(\mathrm{OR}=1.21) \text { and } \\
\text { better for the environment } \\
(\mathrm{OR}=0.87) \text { predicted action } \\
\text { state for willingness to } \\
\text { reduce meat consumption, } \\
\text { all } p<0.001 \text {; age and } \\
\text { education were n.s. }\end{array}$ \\
\hline $\begin{array}{l}\text { Truelove et al., } \\
2012 \text { [26] }\end{array}$ & $\begin{array}{l}\text { Online survey } \\
\text { with open } \\
\text { ended } \\
\text { questions and } \\
\text { behavior } \\
\text { ratings.; } 2008\end{array}$ & $\begin{array}{l}\text { USA; } \\
\text { Undergraduate psychology } \\
\text { majors }(N=112) \text { ( } 69 \text { women } \\
\text { and } 43 \text { men) }\end{array}$ & $\begin{array}{l}\text { Students } \\
\text { perceptions of the } \\
\text { relative impact } \\
\text { and effectiveness } \\
\text { of certain } \\
\text { behaviors on } \\
\text { global warming. }\end{array}$ & $\begin{array}{l}\text { No prior info } \\
\text { given. }\end{array}$ & $\begin{array}{l}\text { Respondents asked to rate } \\
\text { their intention to perform } \\
20 \text { different } \\
\text { proenvironmental } \\
\text { behaviors. 1 (Strongly } \\
\text { unlikely) to } 7 \text { (Strongly } \\
\text { likely) }\end{array}$ & $\begin{array}{l}\text { Reduce your meat } \\
\text { consumption: } 2.99 / 7 \\
\text { SD:2.07 }\end{array}$ & $\begin{array}{l}\text { Effectiveness knowledge } \\
\text { did not significantly } \\
\text { correlate with intention to } \\
\text { perform } \\
\text { behaviors that mitigate GW. } \\
\text { Effectiveness belief did } \\
\text { significantly correlate with } \\
\text { the intention to reduce meat } \\
\text { consumption. }\end{array}$ \\
\hline
\end{tabular}


Table A2. Cont.

\begin{tabular}{|c|c|c|c|c|c|c|c|}
\hline \multirow[b]{2}{*}{$\begin{array}{l}\text { Author(s), } \\
\text { Year }\end{array}$} & \multirow[b]{2}{*}{$\begin{array}{l}\text { Design; Year } \\
\text { Data Collected }\end{array}$} & \multirow[b]{2}{*}{ Country; Sample } & \multirow[b]{2}{*}{$\begin{array}{l}\text { Main Research } \\
\text { Question }\end{array}$} & \multirow[b]{2}{*}{$\begin{array}{l}\text { Provided } \\
\text { Information Prior } \\
\text { the Experiment }\end{array}$} & \multicolumn{3}{|c|}{ Outcome Measure: Willingness to Reduce/Replace } \\
\hline & & & & & $\begin{array}{c}\text { Question or Dependent } \\
\text { Variable }\end{array}$ & Response or Finding & Effect of Covariates \\
\hline $\begin{array}{l}\text { Vanhonacker } \\
\text { et al., } 2013 \\
{[23]^{*}}\end{array}$ & $\begin{array}{l}\text { Online survey; } \\
2011\end{array}$ & $\begin{array}{l}\text { Belgium (Flanders); } \\
\text { convenience sample, } \\
N=221,36 \% \text { men }\end{array}$ & $\begin{array}{l}\text { Attitudes towards } \\
\text { more sustainable } \\
\text { food choices and } \\
\text { consumer } \\
\text { segmentation } \\
\text { based on their } \\
\text { self-evaluated } \\
\text { ecological } \\
\text { footprint. }\end{array}$ & $\begin{array}{l}\text { Explanation of the } \\
\text { concept 'ecological } \\
\text { footprint' }\end{array}$ & $\begin{array}{l}\text { Willingness to reduce } \\
\text { meat consumption } \\
(1=\text { strongly disagree to } \\
5=\text { strongly agree })\end{array}$ & $\begin{array}{l}\text { Meat reduction was rated } \\
\text { the most appealing option } \\
\text { (approx. } \mathrm{M}=3.9 \text {, only bar } \\
\text { chart shown) out of various } \\
\text { options to improve } \\
\text { sustainability of food } \\
\text { choices (e.g., insects, meat } \\
\text { substitutes) }\end{array}$ & n.a. \\
\hline EU Report [35] & $\begin{array}{l}\text { Telephone } \\
\text { survey. } \\
2012\end{array}$ & $\begin{array}{l}27 \text { EUnion countries; aged } \\
15 \text { and above. In each } \\
\text { household, the respondent } \\
\text { was drawn at random } \\
\text { following the "last birthday } \\
\text { rule". } 1000 \text { people sample } \\
\text { per country. Small countries: } \\
500 \text { people sample. }\end{array}$ & $\begin{array}{l}\text { EU citizens' } \\
\text { knowledge of } \\
\text { green products } \\
\text { and their reasons } \\
\text { for buying, or not } \\
\text { buying, } \\
\text { environmentally- } \\
\text { friendly products }\end{array}$ & $\begin{array}{l}\text { The interviewer } \\
\text { read out: "Some } \\
\text { people say large } \\
\text { scale meat } \\
\text { production has a } \\
\text { negative impact on } \\
\text { the environment" }\end{array}$ & $\begin{array}{l}\text { Would you be willing to } \\
\text { do the following for } \\
\text { environmental reasons? } \\
\text { (a) Eat less meat but of } \\
\text { certified origin } \\
\text { (b) Replace most of the } \\
\text { meat you eat by } \\
\text { vegetables }\end{array}$ & $\begin{array}{l}\text { (a) } 80 \% \text { EU citizens willing } \\
\text { to eat less meat but of } \\
\text { certified origin } \\
\text { Highest: Portugal }(89 \%) \\
\text { Lowest: Estonia }(40 \%) \\
\text { (b) } 50 \% \text { EU citizens willing } \\
\text { to replace most of the meat } \\
\text { they eat with vegetables } \\
\text { Highest: Romania }(69 \%) \\
\text { Lowest: The Netherlands } \\
\text { ( } 29 \%) \\
\text { (Information by country can } \\
\text { be found in the report) }\end{array}$ & $\begin{array}{l}\text { The strongest } \\
\text { socio-demographic factor } \\
\text { linked to willingness to } \\
\text { change one's meat } \\
\text { consumption is gender. } \\
\text { Female respondents are } \\
\text { considerably more willing } \\
\text { than male respondents to } \\
\text { replace most of the meat } \\
\text { they eat with vegetables } \\
\text { (59\% and } 40 \% \text {, respectively). } \\
\text { Women are also more } \\
\text { willing to replace beef or } \\
\text { pork with poultry or fish } \\
\text { ( } 76 \% \text { versus } 67 \% \text { ) and eat } \\
\text { less meat but of certified } \\
\text { origin }(83 \% \text { versus } 76 \%) .\end{array}$ \\
\hline
\end{tabular}

Notes n.a.: not assessed; $\mathrm{M}$ = arithmetic mean; $\mathrm{SD}$ = standard deviation; $\mathrm{SES}$ = socioeconomic status. *: As reported by [55]. 


\section{Appendix C}

Table A3. Vegans, vegetarians, and meat consumption curtailers for environmental reasons.

\begin{tabular}{|c|c|c|c|c|c|c|c|}
\hline \multicolumn{8}{|c|}{ Outcome Measure: Reason to Reduce Meat or Become Vegetarian } \\
\hline $\begin{array}{l}\text { Author(s), } \\
\text { Year }\end{array}$ & $\begin{array}{l}\text { Design; Year } \\
\text { Data Collected }\end{array}$ & Country; Sample & $\begin{array}{l}\text { Main Research } \\
\text { Question }\end{array}$ & $\begin{array}{l}\text { Provided } \\
\text { Information Prior } \\
\text { the Experiment }\end{array}$ & $\begin{array}{c}\text { Question or Dependent } \\
\text { Variable }\end{array}$ & Response or Finding & Effect of Covariates \\
\hline $\begin{array}{l}\text { De Backer, } \\
\text { Charlotte J.S. } \\
\text { Hudders, } \\
\text { Liselot; } 2014 \\
\text { [49] }\end{array}$ & $\begin{array}{l}\text { Large-scale } \\
\text { Online survey; } \\
\text { year not } \\
\text { specified. }\end{array}$ & $\begin{array}{l}\text { Belgium; } \\
N=1566 \text { ( } 76 \% \text { women) } \\
\mathrm{M} \text { age = } 26.12 \mathrm{SD}=8.92 \\
10.6 \%=\text { vegetarians; } 41.8 \% \\
\text { semi-vegetarians; } 47.6 \% \\
\text { light-semi-vegetarians. }\end{array}$ & $\begin{array}{l}\text { Motives } \\
\text { underlying the } \\
\text { different forms of } \\
\text { vegetarianism and } \\
\text { semi-vegetarianism } \\
\text { in a culture } \\
\text { where meat } \\
\text { continues to play a } \\
\text { crucial role in } \\
\text { people's diets. }\end{array}$ & $\begin{array}{l}\text { No prior info } \\
\text { provided. }\end{array}$ & $\begin{array}{l}\text { Agree or disagree with a } \\
\text { 7-point Likert scale with } \\
\text { motives for meat } \\
\text { reduction/avoidance. } \\
\text { Ecological motives: } \\
\text { "I don't eat meat every } \\
\text { day because it is better for } \\
\text { the environment," and } \\
\text { "I don't eat meat every } \\
\text { day because eating meat } \\
\text { increases my ecological } \\
\text { footprint". }\end{array}$ & $\begin{array}{l}\text { 143/165 vegetarians } \\
\text { strongly agreed with } \\
\text { ecological motives ( } 6.1 \text { or } \\
\text { higher in a Likert scale } 1-7) \text {. } \\
\text { For } 28 / 143 \text { ecological } \\
\text { concerns were the main } \\
\text { drive (mean of } 6.5 / 7 \text { Liker } \\
\text { scale) The rest of the } \\
\text { vegetarians ( } n=22 \text { ) } \\
\text { disagreed with the } \\
\text { ecological concerns (mean } \\
\text { of } 2.61 / 7 \text { Likert scale). } \\
\text { 323/650 semi-vegetarians: } \\
\text { reported ecological } \\
\text { concerns as the main } \\
\text { motivator for strongly } \\
\text { reducing meat. (Mean of } \\
5.57 / 7 \text { Likert scale) } \\
\text { 254/741 light } \\
\text { semi-vegetarians reported } \\
\text { ecological concerns as the } \\
\text { main motivator for } \\
\text { avoiding meat one or two } \\
\text { days a week. (Mean of } \\
5.12 / 7 \text { Likert scale) }\end{array}$ & $\begin{array}{l}\text { Ecological concern } \\
\text { positively associated with } \\
\text { meat reduction, except for } \\
\text { light semi-vegetarians. }\end{array}$ \\
\hline
\end{tabular}


Table A3. Cont.

\begin{tabular}{|c|c|c|c|c|c|c|c|}
\hline \multirow[b]{2}{*}{$\begin{array}{l}\text { Author(s), } \\
\text { Year }\end{array}$} & \multirow[b]{2}{*}{$\begin{array}{l}\text { Design; Year } \\
\text { Data Collected }\end{array}$} & \multirow[b]{2}{*}{ Country; Sample } & \multirow[b]{2}{*}{$\begin{array}{l}\text { Main Research } \\
\text { Question }\end{array}$} & \multirow[b]{2}{*}{$\begin{array}{l}\text { Provided } \\
\text { Information Prior } \\
\text { the Experiment }\end{array}$} & \multicolumn{3}{|c|}{ Outcome Measure: Reason to Reduce Meat or Become Vegetarian } \\
\hline & & & & & $\begin{array}{c}\text { Question or Dependent } \\
\text { Variable }\end{array}$ & Response or Finding & Effect of Covariates \\
\hline $\begin{array}{l}\text { De Boer et al., } \\
2017 \text { [52] }\end{array}$ & $\begin{array}{l}\text { Face-to-face } \\
\text { interviews; } 2013\end{array}$ & $\begin{array}{l}\text { Netherlands; } \\
\text { two samples of adults (aged } \\
18-35) \\
\text { Native Dutch, } n=357, \\
\text { (Men } 48 \% \text { ) } \\
\text { Second generation Chinese } \\
\text { Dutch, } n=350 \text { (Men } 47 \% \text { ) } \\
\text { Participants were } \\
\text { categorized in four dietary } \\
\text { groups (all self-declared) } \\
\text { (1) Vegetarians } \\
\text { (2) Low meat eaters } \\
\text { (2-3 days a week) } \\
\text { (3) Medium meat eaters } \\
\text { (4-5 days a week) } \\
\text { (4) High meat eaters (6 days } \\
\text { or more) }\end{array}$ & $\begin{array}{l}\text { Differences } \\
\text { between } \\
\text { vegetarians and } \\
\text { three categories of } \\
\text { meat eaters in } \\
\text { relation to (1) key } \\
\text { characteristics of } \\
\text { their hot meal, } \\
\text { (2) strength and } \\
\text { profile of their } \\
\text { food-related } \\
\text { motivation, } \\
\text { and (3) reasons for } \\
\text { and reasons } \\
\text { against frequently } \\
\text { eating meat? }\end{array}$ & $\begin{array}{l}\text { No prior info } \\
\text { provided. }\end{array}$ & $\begin{array}{l}\text { Indicate three reasons for } \\
\text { not frequently eating } \\
\text { meat. Among them, } \\
\text { participants could choose } \\
\text { "Because it's better for the } \\
\text { environment". }\end{array}$ & $\begin{array}{l}\text { NATIVE DUTCH; } \\
\text { Self-declared vegetarians: } \\
21 \% \text { indicated the } \\
\text { environment as a reason for } \\
\text { not frequently eating meat. } \\
\text { Low meat-eaters: } 30 \% \\
\text { Medium meat-eaters: } 44 \% \\
\text { High meat-eaters: } 41 \% \\
\text { TOTAL: } 38 \% \\
\text { CHINESE DUTCH: } \\
\text { Self-declared vegetarians: } \\
42 \% \\
\text { Low meat-eaters: } 38 \% \\
\text { Medium meat-eaters: } 32 \% \\
\text { High meat-eaters: } 15 \% \\
\text { TOTAL: } 26 \% \\
\text { Environmental and } \\
\text { financial reasons were } \\
\text { mentioned relatively often, } \\
\text { but according to the } \\
\text { authors, the fact that they } \\
\text { were also mentioned by } \\
\text { high meat-eaters indicates } \\
\text { that, under the current } \\
\text { circumstances, these } \\
\text { reasons are not decisive for } \\
\text { a reduction in meat } \\
\text { consumption. }\end{array}$ & $\begin{array}{l}\text { Native Dutch: the more } \\
\text { meat they eat, the more they } \\
\text { would give an } \\
\text { environmental reason for } \\
\text { not eating meat. } \\
\text { Chinese Dutch, the less } \\
\text { meat they eat, the more } \\
\text { report the environment as } \\
\text { reason for not eating meat. } \\
\text { In both samples, the } \\
\text { vegetarians were more often } \\
\text { women (about } 70 \% \text {, } \\
\text { whereas the high } \\
\text { meat-eaters were more } \\
\text { often men (about } 70 \%) \text {. }\end{array}$ \\
\hline
\end{tabular}


Table A3. Cont.

\begin{tabular}{|c|c|c|c|c|c|c|c|}
\hline \multirow[b]{2}{*}{$\begin{array}{l}\text { Author(s), } \\
\text { Year }\end{array}$} & \multirow[b]{2}{*}{$\begin{array}{l}\text { Design; Year } \\
\text { Data Collected }\end{array}$} & \multirow[b]{2}{*}{ Country; Sample } & \multirow[b]{2}{*}{$\begin{array}{l}\text { Main Research } \\
\text { Question }\end{array}$} & \multirow[b]{2}{*}{$\begin{array}{l}\text { Provided } \\
\text { Information Prior } \\
\text { the Experiment }\end{array}$} & \multicolumn{3}{|c|}{ Outcome Measure: Reason to Reduce Meat or Become Vegetarian } \\
\hline & & & & & $\begin{array}{l}\text { Question or Dependent } \\
\text { Variable }\end{array}$ & Response or Finding & Effect of Covariates \\
\hline $\begin{array}{l}\text { Dyett, Patricia } \\
\text { A., et al., } 2013 \\
\text { [40] }\end{array}$ & $\begin{array}{l}\text { Postal survey; } \\
\text { (year not } \\
\text { reported) }\end{array}$ & $\begin{array}{l}\text { United States; } \\
N=100 \\
\text { Population of self-reported } \\
\text { vegans for more than } 9 \\
\text { months living in different } \\
\text { U.S. States. } \\
\text { Age: } 25-75 \text { yrs old } \\
\text { Vegans defined as } \\
\text { individuals who used no } \\
\text { meat, fish, or poultry, and } \\
\text { who used dairy- or } \\
\text { egg-containing products } \\
\text { less than } \\
\text { once per month. }\end{array}$ & $\begin{array}{l}\text { Discover the main } \\
\text { reasons for } \\
\text { adopting and } \\
\text { maintaining a } \\
\text { vegan lifestyle and } \\
\text { to determine } \\
\text { whether } \\
\text { participants' diet } \\
\text { and lifestyle } \\
\text { choices coincided } \\
\text { with positive } \\
\text { health indices and } \\
\text { selected outcome } \\
\text { assessment. }\end{array}$ & $\begin{array}{l}\text { No prior info } \\
\text { provided. }\end{array}$ & Reason for being vegan & $\begin{array}{l}\text { Because environmental } \\
\text { values }(2 \%)\end{array}$ & n.a. \\
\hline $\begin{array}{l}\text { Turner-McGriev } \\
\text { G. et al., } 2016 \\
\text { [41] }\end{array}$ & $\begin{array}{l}\text { online quota } \\
\text { survey; year not } \\
\text { specified; }\end{array}$ & $\begin{array}{l}\text { Majority }(90 \%) \text { from the } \\
\text { United States; } \\
N=422(n=125 \text { ULTRA, } \\
n=152 \text { FULL, } n=145 \\
\text { HALF) More ULTRA } \\
\text { participants were men (63\%) } \\
\text { (vs. FULL ( } 37 \% \text { ) and HALF } \\
(23 \%)\end{array}$ & $\begin{array}{l}\text { Examine } \\
\text { differences in } \\
\text { current vegetarian } \\
\text { and vegan diets, } \\
\text { reasons for it and } \\
\text { other dietary } \\
\text { behaviors among } \\
\text { long distance } \\
\text { runners. }\end{array}$ & $\begin{array}{l}\text { No prior info } \\
\text { provided. }\end{array}$ & $\begin{array}{l}\text { Participants asked to } \\
\text { select all reasons for } \\
\text { choosing their current diet } \\
\text { that apply to them from a } \\
\text { list of } 12 \text { reasons } \\
\text { (including an option to } \\
\text { select no reason or to } \\
\text { write in an answer). }\end{array}$ & $\begin{array}{l}\text { More ULTRA participants } \\
(n=25,20 \%) \text { reported that } \\
\text { environmental concerns } \\
\text { shaped their diet choice as } \\
\text { compared with FULL and } \\
\text { HALF participants }(n=36, \\
\left.12 \% ; \chi^{2}=4.4, p=0.04\right) .\end{array}$ & n.a. \\
\hline
\end{tabular}


Table A3. Cont.

\begin{tabular}{|c|c|c|c|c|c|c|c|}
\hline \multirow[b]{2}{*}{$\begin{array}{l}\text { Author(s), } \\
\text { Year }\end{array}$} & \multirow[b]{2}{*}{$\begin{array}{l}\text { Design; Year } \\
\text { Data Collected }\end{array}$} & \multirow[b]{2}{*}{ Country; Sample } & \multirow[b]{2}{*}{$\begin{array}{l}\text { Main Research } \\
\text { Question }\end{array}$} & \multirow[b]{2}{*}{$\begin{array}{l}\text { Provided } \\
\text { Information Prior } \\
\text { the Experiment }\end{array}$} & \multicolumn{3}{|c|}{ Outcome Measure: Reason to Reduce Meat or Become Vegetarian } \\
\hline & & & & & $\begin{array}{c}\text { Question or Dependent } \\
\text { Variable }\end{array}$ & Response or Finding & Effect of Covariates \\
\hline $\begin{array}{l}\text { Haverstock, } \\
\text { Katie, et al., } \\
2012 \text { [48] }\end{array}$ & $\begin{array}{l}\text { Food Choice } \\
\text { Questionnaire; } \\
\text { year not } \\
\text { specified; }\end{array}$ & $\begin{array}{l}\text { International online sample; } \\
N=247 \text { ( } 196=\text { current } \\
\text { animal product limiters and } \\
51 \text { former limiters) } \\
211=\text { females; } \\
\text { Age = } 18 \text { to } 66(\mathrm{M}=29.05 \\
\mathrm{SD}=9.39) \\
222=\text { Euro-Americans. }\end{array}$ & $\begin{array}{l}\text { Similarities and } \\
\text { differences } \\
\text { between current } \\
\text { and former animal } \\
\text { product limiters. }\end{array}$ & $\begin{array}{l}\text { No prior info } \\
\text { provided. }\end{array}$ & $\begin{array}{l}\text { Eight items concerning } \\
\text { ethical food choice } \\
\text { motives were also } \\
\text { included [...] These ethical } \\
\text { motives include animal } \\
\text { welfare, environmental } \\
\text { protection, political } \\
\text { values, and religion. } \\
\text { Likert scale: } 1=\text { not } \\
\text { important to } 4=\text { very } \\
\text { important. }\end{array}$ & $\begin{array}{l}\text { Importance given to } \\
\text { environmental reasons to } \\
\text { reduce or avoid meat. } \\
\text { CURRENT LIMITERS: } \\
\text { Vegans }(n=119) \mathrm{M}=3.10, \\
\mathrm{SD}=0.68 \\
\text { Vegetarian }(n=54) \mathrm{M}=2.71 \text {, } \\
\mathrm{SD}=0.74 \\
\text { Pescatarian }(n=22) \\
\mathrm{M}=2.79, \mathrm{SD}=0.75 \\
\text { FORMER LIMITERS: } \\
\text { Now a regular meat eater } \\
(n=16) \\
\mathrm{M}=2.13, \mathrm{SD}=0.94 \\
\text { Now a occasional meat } \\
\text { eater }(n=26) \\
\mathrm{M}=2.67, \mathrm{SD}=0.80 \\
\text { Now a meat avoider }(n=4) \\
\mathrm{M}=2.17, \mathrm{SD}=0.43 \\
\text { Now a pescatarian }(n=5) \\
\mathrm{M}=2.54, \mathrm{SD}=0.88\end{array}$ & $\begin{array}{l}\text { Few gender differences. } \\
\text { Women more strongly } \\
\text { endorsed health and the } \\
\text { environment motives than } \\
\text { did men. }\end{array}$ \\
\hline
\end{tabular}


Table A3. Cont.

\begin{tabular}{|c|c|c|c|c|c|c|c|}
\hline \multirow[b]{2}{*}{$\begin{array}{l}\text { Author(s), } \\
\text { Year }\end{array}$} & \multirow[b]{2}{*}{$\begin{array}{l}\text { Design; Year } \\
\text { Data Collected }\end{array}$} & \multirow[b]{2}{*}{ Country; Sample } & \multirow[b]{2}{*}{$\begin{array}{l}\text { Main Research } \\
\text { Question }\end{array}$} & \multirow[b]{2}{*}{$\begin{array}{l}\text { Provided } \\
\text { Information Prior } \\
\text { the Experiment }\end{array}$} & \multicolumn{3}{|c|}{ Outcome Measure: Reason to Reduce Meat or Become Vegetarian } \\
\hline & & & & & $\begin{array}{c}\text { Question or Dependent } \\
\text { Variable }\end{array}$ & Response or Finding & Effect of Covariates \\
\hline $\begin{array}{l}\text { Hoffman, } \\
\text { Sarah R. } \\
\text { et al., 2013 [39] }\end{array}$ & $\begin{array}{l}\text { Online survey; } \\
2011\end{array}$ & $\begin{array}{l}\text { USA; } \\
\text { People recruited through } \\
\text { Facebook, Google, and } \\
\text { vegetarian dedicated } \\
\text { webpages. } N=312 \\
\text { Age: } 18-69 .(42 \%=\text { age } \\
20-29) 15.4 \% \text { men, } 84.6 \% \\
\text { women. } 68.3 \% \text { had some } \\
\text { form of Higher Education. } \\
86.5 \% \text { White-Caucasian } \\
56.7 \% \text { had an income of } \\
<49,000 \text { USD } \\
\text { Vegetarian } 49.4 \text { (vegetarian) } \\
\text { and } 50.6 \text { (vegan). }\end{array}$ & $\begin{array}{l}\text { Examine the } \\
\text { differences } \\
\text { between health } \\
\text { and ethical } \\
\text { vegetarians by } \\
\text { comparing } \\
\text { conviction, } \\
\text { nutrition } \\
\text { knowledge, } \\
\text { dietary restriction, } \\
\text { and years as } \\
\text { vegetarian } \\
\text { between the two } \\
\text { groups. }\end{array}$ & $\begin{array}{l}\text { No prior info } \\
\text { provided. }\end{array}$ & $\begin{array}{l}\text { In order to place subjects } \\
\text { into categories (i.e., health, } \\
\text { ethical, or other), two } \\
\text { multiple choice items } \\
\text { were created: "The main } \\
\text { reason I became a } \\
\text { vegetarian was because of } \\
\text { (check only one)," "The } \\
\text { main reason I am (still) a } \\
\text { vegetarian is because of } \\
\text { (check only one)." } \\
\text { Fourteen options were } \\
\text { given in addition to the } \\
\text { option "other" }\end{array}$ & $\begin{array}{l}234=\text { ethical reasons } \\
(\text { animal, ethics, religion, } \\
\text { environment) }(10=\text { the } \\
\text { environment) as initial } \\
\text { reason to become } \\
\text { vegetarian. }\end{array}$ & Not reported. \\
\hline $\begin{array}{l}\text { Izmirli, et al., } \\
2011 \text { [51] }\end{array}$ & $\begin{array}{l}\text { Survey; year } \\
\text { not specified; }\end{array}$ & $\begin{array}{l}11 \text { Eurasian countries; } \\
N=3433 \text { university } \\
\text { students from } 103 \\
\text { universities. } \\
47 \% \text { avoided some meat } \\
\text { products. } \\
4 \% \text { vegetarians } \\
0.4 \% \text { vegans }\end{array}$ & $\begin{array}{l}\text { Determine the } \\
\text { relationship } \\
\text { between the } \\
\text { consumption of } \\
\text { animal products } \\
\text { and attitudes } \\
\text { towards animals } \\
\text { among university } \\
\text { students in } \\
\text { Eurasia }\end{array}$ & $\begin{array}{l}\text { No prior info } \\
\text { provided. }\end{array}$ & $\begin{array}{l}\text { Specify the major reason } \\
\text { for meat avoidance like } \\
\text { health concerns, religious } \\
\text { instruction, concerns for } \\
\text { the suffering of animals or } \\
\text { for the environment. }\end{array}$ & $\begin{array}{l}479 \text { students }(38.1 \%) \text { gave } \\
\text { the environmental reason. }\end{array}$ & $\begin{array}{l}\text { Among "some meat } \\
\text { avoidants" (total = } 1147) \\
46841 \% \text { because of the } \\
\text { environment. (Most chosen } \\
\text { reason). Among } \\
\text { "vegetarians" (total = 99) } \\
9(9 \%) \text { because of the } \\
\text { environment. } \\
\text { Among "vegans" (total = 7) } \\
2(29 \%) \text { because of the } \\
\text { environment. }\end{array}$ \\
\hline
\end{tabular}


Table A3. Cont.

\begin{tabular}{|c|c|c|c|c|c|c|c|}
\hline \multirow[b]{2}{*}{$\begin{array}{l}\text { Author(s), } \\
\text { Year }\end{array}$} & \multirow[b]{2}{*}{$\begin{array}{l}\text { Design; Year } \\
\text { Data Collected }\end{array}$} & \multirow[b]{2}{*}{ Country; Sample } & \multirow[b]{2}{*}{$\begin{array}{l}\text { Main Research } \\
\text { Question }\end{array}$} & \multirow[b]{2}{*}{$\begin{array}{l}\text { Provided } \\
\text { Information Prior } \\
\text { the Experiment }\end{array}$} & \multicolumn{3}{|c|}{ Outcome Measure: Reason to Reduce Meat or Become Vegetarian } \\
\hline & & & & & $\begin{array}{l}\text { Question or Dependent } \\
\text { Variable }\end{array}$ & Response or Finding & Effect of Covariates \\
\hline $\begin{array}{l}\text { Lindeman, } \\
\text { Marjaana, } \\
\text { et al., 2001 [44] }\end{array}$ & $\begin{array}{l}\text { STUDY 1 } \\
\text { Food Choice } \\
\text { Questionnaire; } \\
\text { year not } \\
\text { specified. }\end{array}$ & $\begin{array}{l}\text { Finland; } \\
82 \text { female participants. Age: } \\
17-3 \text { years old. } 30.4 \% \\
\text { semi-vegetarians and } 25.3 \% \\
\text { vegetarians. }\end{array}$ & $\begin{array}{l}\text { The construction } \\
\text { of food choice } \\
\text { ideologies and the } \\
\text { ways dietary } \\
\text { groups endorse } \\
\text { them. }\end{array}$ & $\begin{array}{l}\text { No prior info } \\
\text { provided. }\end{array}$ & $\begin{array}{l}\text { Food Choice } \\
\text { Questionnaire. Motives } \\
\text { assessed among others: } \\
\text { ecological welfare } \\
\text { (including animal welfare } \\
\text { and protection of nature). } \\
\text { Subjects had to rate the } \\
\text { statement "It is important } \\
\text { to me that the food I eat } \\
\text { on a typical day..." on a } \\
\text { 4-point scale (1= not at all } \\
\text { important, } 4=\text { very } \\
\text { important). }\end{array}$ & $\begin{array}{l}\text { Ecological welfare. } \\
\text { Semi and full vegetarians: } \\
M=3, S D=0.74\end{array}$ & $\begin{array}{l}\text { Vegetarians regarded } \\
\text { ecological food choice } \\
\text { reasons as more important } \\
\text { than semivegetarians did, } \\
t(45)=-4.12, p<0.001 \text {. }\end{array}$ \\
\hline $\begin{array}{l}\text { Lindeman, } \\
\text { Marjaana, } \\
\text { et al., } 2001\end{array}$ & $\begin{array}{l}\text { STUDY } 2 \\
\text { Food Choice } \\
\text { Questionnaire; } \\
\text { year not } \\
\text { specified. }\end{array}$ & $\begin{array}{l}\text { Finland; } \\
N=149 \text { women. } \\
\text { Age: } 19-74 \text { Mean age: } 31.5 . \\
44.3 \text { full time students. } \\
41.6 \% \text { employed women. } \\
16.8 \% \text { semivegetarians and } \\
10.7 \% \text { vegetarians. }\end{array}$ & Idem & $\begin{array}{l}\text { No prior info } \\
\text { provided. }\end{array}$ & Idem & $\begin{array}{l}\text { Ecological welfare. } \\
\text { Semi and full vegetarians: } \\
\mathrm{M}=2.94, \mathrm{SD}=0.80\end{array}$ & n.a. \\
\hline
\end{tabular}


Table A3. Cont.

\begin{tabular}{|c|c|c|c|c|c|c|c|}
\hline \multirow[b]{2}{*}{$\begin{array}{l}\text { Author(s), } \\
\text { Year }\end{array}$} & \multirow[b]{2}{*}{$\begin{array}{l}\text { Design; Year } \\
\text { Data Collected }\end{array}$} & \multirow[b]{2}{*}{ Country; Sample } & \multirow[b]{2}{*}{$\begin{array}{l}\text { Main Research } \\
\text { Question }\end{array}$} & \multirow[b]{2}{*}{$\begin{array}{l}\text { Provided } \\
\text { Information Prior } \\
\text { the Experiment }\end{array}$} & \multicolumn{3}{|c|}{ Outcome Measure: Reason to Reduce Meat or Become Vegetarian } \\
\hline & & & & & $\begin{array}{c}\text { Question or Dependent } \\
\text { Variable }\end{array}$ & Response or Finding & Effect of Covariates \\
\hline $\begin{array}{l}\text { Péneu, et al., } \\
2017 \text { [50] }\end{array}$ & $\begin{array}{l}\text { Online survey. } \\
\text { Ongoing } \\
\text { web-based } \\
\text { prospective } \\
\text { observational } \\
\text { cohort study } \\
\text { launched in } \\
\text { France in May } \\
2009 \text { with a } \\
\text { scheduled } \\
\text { follow-up of } 10 \\
\text { years. }\end{array}$ & $\begin{array}{l}\text { France; } \\
N=22,935 \\
\text { (5688 men) }\end{array}$ & $\begin{array}{l}\text { Investigate the } \\
\text { sociodemographic } \\
\text { profiles of } \\
\text { individuals } \\
\text { reporting health } \\
\text { and environmental } \\
\text { dilemmas when } \\
\text { purchasing meat, } \\
\text { fish and dairy } \\
\text { products, and } \\
\text { compare diet } \\
\text { quality of } \\
\text { individuals with } \\
\text { and without } \\
\text { dilemma. }\end{array}$ & $\begin{array}{l}\text { No prior info } \\
\text { provided. }\end{array}$ & $\begin{array}{l}\text { Respondents have to } \\
\text { agree or disagree with the } \\
\text { following statement: } \\
\text { "I avoid purchasing } \\
\text { [meat/fish/dairy } \\
\text { products] for } \\
\text { environmental issues" }\end{array}$ & $25 \%$ strongly agree or agree & \\
\hline $\begin{array}{l}\text { Péneu, et al., } \\
2017\end{array}$ & & & & & $\begin{array}{l}\text { Asked to agree or not } \\
\text { with "I am torn between } \\
\text { purchasing } \\
\text { [meat/fish/dairy } \\
\text { products] to follow } \\
\text { dietary guidelines or limit } \\
\text { purchase for } \\
\text { environmental issues". }\end{array}$ & $31.94 \%$ said YES & $\begin{array}{l}\text { - Women declared more } \\
\text { dilemma in the case of meat } \\
\text { than men. } \\
\text { - In the case of meat, } \\
\text { individuals with greater } \\
\text { educational level and } \\
\text { household including only } \\
\text { one adult were more likely } \\
\text { to report a dilemma. }\end{array}$ \\
\hline
\end{tabular}


Table A3. Cont.

\begin{tabular}{|c|c|c|c|c|c|c|c|}
\hline \multirow[b]{2}{*}{$\begin{array}{l}\text { Author(s), } \\
\text { Year }\end{array}$} & \multirow[b]{2}{*}{$\begin{array}{l}\text { Design; Year } \\
\text { Data Collected }\end{array}$} & \multirow[b]{2}{*}{ Country; Sample } & \multirow[b]{2}{*}{$\begin{array}{l}\text { Main Research } \\
\text { Question }\end{array}$} & \multirow[b]{2}{*}{$\begin{array}{l}\text { Provided } \\
\text { Information Prior } \\
\text { the Experiment }\end{array}$} & \multicolumn{3}{|c|}{ Outcome Measure: Reason to Reduce Meat or Become Vegetarian } \\
\hline & & & & & $\begin{array}{l}\text { Question or Dependent } \\
\text { Variable }\end{array}$ & Response or Finding & Effect of Covariates \\
\hline $\begin{array}{l}\text { Povey et al., } \\
2001 \text { [53] }\end{array}$ & $\begin{array}{l}\text { Open ended } \\
\text { questionnaires; } \\
\text { year not } \\
\text { reported. }\end{array}$ & $\begin{array}{l}\text { United Kingdom; } \\
\text { Convenience sample; } \\
111 \text { respondents ( } 25 \text { meat } \\
\text { eaters, } 26 \text { meat avoiders, } 34 \\
\text { vegetarians, } 26 \text { vegans). }\end{array}$ & $\begin{array}{l}\text { Examine } \\
\text { differences } \\
\text { between the } \\
\text { attitudes and } \\
\text { beliefs of four } \\
\text { dietary groups } \\
\text { (meat eaters, meat } \\
\text { avoiders, } \\
\text { vegetarians and } \\
\text { vegans) and the } \\
\text { extent to which } \\
\text { attitudes } \\
\text { influenced } \\
\text { intentions to } \\
\text { follow a diet. }\end{array}$ & $\begin{array}{l}\text { No prior info } \\
\text { provided. }\end{array}$ & $\begin{array}{l}\text { Record salient thoughts, } \\
\text { beliefs and feelings } \\
\text { towards these three diets: } \\
\text { meat, vegetarian and } \\
\text { vegan. A maximum of } \\
\text { eight thoughts, beliefs or } \\
\text { feelings could be recorded } \\
\text { by participants. }\end{array}$ & $\begin{array}{l}\text { MEAT DIET: } \\
6 / 26 \text { vegans and } 6 / 26 \text { meat } \\
\text { avoiders named } \\
\text { environmental problems as } \\
\text { a salient belief towards } \\
\text { eating a meat diet. } \\
\text { VEGETARIAN DIET: } \\
4 / 26 \text { vegans mentioned a } \\
\text { vegetarian diet to be } \\
\text { environmentally friendly. } \\
\text { VEGAN DIET: } \\
12 / 26 \text { vegans mentioned it } \\
\text { to be environmentally } \\
\text { friendly. }\end{array}$ & n.a. \\
\hline $\begin{array}{l}\text { Pribis, et al., } \\
2010 \text { [43] }\end{array}$ & $\begin{array}{l}\text { cross-sectional, } \\
\text { observational } \\
\text { study; } 2007\end{array}$ & $\begin{array}{l}\text { United States; } \\
\text { Andrews University (SDA } \\
\text { institution) undergraduate } \\
\text { students and their } \\
\text { respective families. } \\
N=609 \text { participants. ( } 35 \% \\
\text { male) } \\
\text { Mean age }=31 \text { years old. } \\
4 \% \text { vegans; } 25 \% \text { lacto-ovo } \\
\text { vegetarians; } 4 \% \\
\text { pesco-vegetarians; } 67 \% \\
\text { non-vegetarians. }\end{array}$ & $\begin{array}{l}\text { Examine whether } \\
\text { reasons to adopt } \\
\text { vegetarian lifestyle } \\
\text { differ significantly } \\
\text { among } \\
\text { generations. }\end{array}$ & $\begin{array}{l}\text { No prior info } \\
\text { provided. }\end{array}$ & $\begin{array}{l}\text { Using a Likert Scale from } \\
1 \text { to } 5 \text { (strongly disagree } \\
\text { [1]-agree [2]-no opinion } \\
\text { [3]-agree [4]-strongly } \\
\text { agree [5]) participants } \\
\text { rated reasons why they } \\
\text { choose a vegetarian } \\
\text { lifestyle. } \\
\text { Vegetarian reason: } \\
\text { "vegetarian lifestyle is } \\
\text { much more protective } \\
\text { against the environment" }\end{array}$ & $\begin{array}{l}\text { Responses across } \\
\text { generations: } \\
\text { 11-20 years old: } 3.95 \\
\text { 21-40 years old: } 3.69 \\
\text { 41-60 years old: } 3.75 \\
61 \text { or older: } 3.79\end{array}$ & $\begin{array}{l}\text { Younger people } \\
\text { (11-20 years) also } \\
\text { significantly agreed more } \\
\text { with the environmental } \\
\text { reason }(p=0.025) .\end{array}$ \\
\hline
\end{tabular}


Table A3. Cont.

\begin{tabular}{|c|c|c|c|c|c|c|c|}
\hline \multirow[b]{2}{*}{$\begin{array}{l}\text { Author(s), } \\
\text { Year }\end{array}$} & \multirow[b]{2}{*}{$\begin{array}{l}\text { Design; Year } \\
\text { Data Collected }\end{array}$} & \multirow[b]{2}{*}{ Country; Sample } & \multirow[b]{2}{*}{$\begin{array}{l}\text { Main Research } \\
\text { Question }\end{array}$} & \multirow[b]{2}{*}{$\begin{array}{l}\text { Provided } \\
\text { Information Prior } \\
\text { the Experiment }\end{array}$} & \multicolumn{3}{|c|}{ Outcome Measure: Reason to Reduce Meat or Become Vegetarian } \\
\hline & & & & & $\begin{array}{c}\text { Question or Dependent } \\
\text { Variable }\end{array}$ & Response or Finding & Effect of Covariates \\
\hline $\begin{array}{l}\text { Rozin, et al., } \\
1997 \text { [45] }\end{array}$ & $\begin{array}{l}\text { Questionnaire; } \\
1987\end{array}$ & $\begin{array}{l}\text { United States; } \\
N=104 \text { self-identified as at } \\
\text { least reluctant to meat. } \\
34=\text { male } \\
\text { Mean age: } 26.6(\mathrm{SD}=8.95)\end{array}$ & $\begin{array}{l}\text { Describe } \\
\text { moralization in the } \\
\text { domain of } \\
\text { vegetarianism. }\end{array}$ & $\begin{array}{l}\text { No prior info } \\
\text { provided. }\end{array}$ & $\begin{array}{l}\text { A list of } 20 \text { possible } \\
\text { reasons for avoiding meat. } \\
\text { Subjects indicated both } \\
\text { current agreement } \\
\text { (5-point scale ranging } \\
\text { from disagree strongly to } \\
\text { agree strongly) with each } \\
\text { reason and, if relevant, the } \\
\text { time of onset of the reason } \\
\text { ("this was your first } \\
\text { reason for avoiding meat," } \\
\text { "this was one of the } \\
\text { earliest reasons for } \\
\text { avoiding meat," "this was } \\
\text { not one of the earliest } \\
\text { reasons for avoiding } \\
\text { meat," or "this was never } \\
\text { a reason for avoiding } \\
\text { meat"). }\end{array}$ & $\begin{array}{l}5.8 \% \text { "initial reason" to } \\
\text { avoid meat. } \\
38.2 \% \text { strongly agreed } \\
(22.5 \% \text { agreed) with } \\
\text { ecological reason as current } \\
\text { reason. }\end{array}$ & n.a. \\
\hline & & & & & $\begin{array}{l}\text { Ecological reason: "I resist } \\
\text { [avoid] eating "meat" } \\
\text { because it is wasteful of } \\
\text { resources to eat animal } \\
\text { rather than vegetable } \\
\text { products, especially in a } \\
\text { world where people are } \\
\text { starving." }\end{array}$ & & \\
\hline
\end{tabular}


Table A3. Cont.

\begin{tabular}{|c|c|c|c|c|c|c|c|}
\hline \multirow[b]{2}{*}{$\begin{array}{l}\text { Author(s), } \\
\text { Year }\end{array}$} & \multirow[b]{2}{*}{$\begin{array}{l}\text { Design; Year } \\
\text { Data Collected }\end{array}$} & \multirow[b]{2}{*}{ Country; Sample } & \multirow[b]{2}{*}{$\begin{array}{l}\text { Main Research } \\
\text { Question }\end{array}$} & \multirow[b]{2}{*}{$\begin{array}{l}\text { Provided } \\
\text { Information Prior } \\
\text { the Experiment }\end{array}$} & \multicolumn{3}{|c|}{ Outcome Measure: Reason to Reduce Meat or Become Vegetarian } \\
\hline & & & & & $\begin{array}{l}\text { Question or Dependent } \\
\text { Variable }\end{array}$ & Response or Finding & Effect of Covariates \\
\hline $\begin{array}{l}\text { Schösler et al., } \\
2015 \text { [36] * }\end{array}$ & $\begin{array}{l}\text { Face-to-face } \\
\text { interview; } 2013\end{array}$ & $\begin{array}{l}\text { Netherlands; } \\
\text { quota samples of second- } \\
\text { generation migrants: } \\
\text { Turkish/Kurdish } N=350, \\
\text { Chinese/Hong Kongese } \\
N=350, \text { Native Dutch } \\
N=357 ; 47-49 \% \text { men }\end{array}$ & $\begin{array}{l}\text { Gender differences } \\
\text { in meat } \\
\text { consumption and } \\
\text { reduction across } \\
\text { ethnic group }\end{array}$ & & $\begin{array}{l}\text { Reasons for not frequently } \\
\text { eating meat (selection of } \\
\text { maximum } 3 \text { reasons out } \\
\text { of } 9 \text { reasons) }\end{array}$ & $\begin{array}{l}\text { It's better for the } \\
\text { environment' was selected } \\
\text { by } 2 \% \text { Turks, } 26 \% \text { Chinese, } \\
38 \% \text { Native Dutch }\end{array}$ & \\
\hline $\begin{array}{l}\text { Study 3: } \\
\text { Schösler, de } \\
\text { Boer, \& } \\
\text { Boersema, } \\
2014 \text { [46] * }\end{array}$ & $\begin{array}{l}\text { Online survey; } \\
2010\end{array}$ & $\begin{array}{l}\text { Netherlands; } \\
\text { quota sample, } N=1083, \\
50 \% \text { men }\end{array}$ & $\begin{array}{l}\text { Cluster analysis } \\
\text { based on type of } \\
\text { eating-related } \\
\text { motivation and } \\
\text { profiling of } \\
\text { segments related } \\
\text { to meat } \\
\text { consumption }\end{array}$ & $\begin{array}{l}\text { No info before } \\
\text { questioning }\end{array}$ & $\begin{array}{l}\text { Reasons for not frequently } \\
\text { eating meat (selection of } \\
\text { maximum } 3 \text { reasons out } \\
\text { of } 9 \text { reasons) }\end{array}$ & $\begin{array}{l}19 \% \text { selected 'It's better for } \\
\text { the environment' }\end{array}$ & $\begin{array}{l}34 \% \text { of those consumers } \\
\text { who internalized the } \\
\text { importance of the } \\
\text { food-nature relationship } \\
\text { agreed that eating less meat } \\
\text { is better for the } \\
\text { environment. }\end{array}$ \\
\hline $\begin{array}{l}\text { Vanhonacker } \\
\text { et al., } 2013 \\
{[23]^{*}}\end{array}$ & $\begin{array}{l}\text { Online survey; } \\
2011\end{array}$ & $\begin{array}{l}\text { Belgium (Flanders); } \\
\text { convenience sample, } \\
N=221,36 \% \text { men }\end{array}$ & $\begin{array}{l}\text { Attitudes towards } \\
\text { more sustainable } \\
\text { food choices and } \\
\text { consumer } \\
\text { segmentation } \\
\text { based on their } \\
\text { self-evaluated } \\
\text { ecological } \\
\text { footprint. }\end{array}$ & $\begin{array}{l}\text { Explanation of the } \\
\text { concept 'ecological } \\
\text { footprint' }\end{array}$ & $\begin{array}{l}\text { Participants had to } \\
\text { indicate } \\
\text { environmentally-friendly } \\
\text { behaviors (what they } \\
\text { actually do) }\end{array}$ & $\begin{array}{l}4 \% \text { consume less meat per } \\
\text { meal to reduce their } \\
\text { ecological footprint }\end{array}$ & n.a. \\
\hline
\end{tabular}


Table A3. Cont.

\begin{tabular}{|c|c|c|c|c|c|c|c|}
\hline \multirow[b]{2}{*}{$\begin{array}{l}\text { Author(s), } \\
\text { Year }\end{array}$} & \multirow[b]{2}{*}{$\begin{array}{l}\text { Design; Year } \\
\text { Data Collected }\end{array}$} & \multirow[b]{2}{*}{ Country; Sample } & \multirow[b]{2}{*}{$\begin{array}{l}\text { Main Research } \\
\text { Question }\end{array}$} & \multirow[b]{2}{*}{$\begin{array}{l}\text { Provided } \\
\text { Information Prior } \\
\text { the Experiment }\end{array}$} & \multicolumn{3}{|c|}{ Outcome Measure: Reason to Reduce Meat or Become Vegetarian } \\
\hline & & & & & $\begin{array}{c}\text { Question or Dependent } \\
\text { Variable }\end{array}$ & Response or Finding & Effect of Covariates \\
\hline $\begin{array}{l}\text { Verain et al., } \\
2015 \text { [47] * }\end{array}$ & $\begin{array}{l}\text { Online survey; } \\
2011\end{array}$ & $\begin{array}{l}\text { Netherlands; } \\
\text { quota sample, } N=942 \\
50 \% \text { men. }\end{array}$ & $\begin{array}{l}\text { Segmentation of } \\
\text { consumers based } \\
\text { on sustainable } \\
\text { food behaviors } \\
\text { and profiling of } \\
\text { segments }\end{array}$ & No info & $\begin{array}{l}\text { Performance of } \\
\text { sustainable food } \\
\text { behaviors at least once a } \\
\text { month in the previous } \\
\text { year ('yes', 'no'). }\end{array}$ & $\begin{array}{l}\text { One meat-free day a week } \\
(56 \%) \text { and smaller meat } \\
\text { portions }(52 \%) \text { were the } \\
\text { most popular sustainable } \\
\text { food behaviors compared } \\
\text { with other behaviors (e.g., } \\
\text { buying organic meat or } \\
\text { dairy) }\end{array}$ & $\begin{array}{l}\text { Female gender }(\beta=0.08, \\
p<0.001), \text { age }(\beta=0.21, \\
p<0.05) \text { and variables on } \\
\text { personal/social norms and } \\
\text { subjective knowledge about } \\
\text { sustainable food choices } \\
\text { positively predicted } \\
\text { curtailment behavior } \\
\text { (average of four items: } \\
\text { eating smaller portions of } \\
\text { meat, eat less diary, eating } \\
\text { less, one meat-free day a } \\
\text { week) }\end{array}$ \\
\hline $\begin{array}{l}\text { White et al. } \\
1999 \text { [42] }\end{array}$ & $\begin{array}{l}\text { Survey; date } \\
\text { not specified }\end{array}$ & $\begin{array}{l}\text { United States; } \\
\text { Random sample. } \\
N=2500 \text { women from each } \\
\text { of the past decades' } \\
\text { graduating medical school } \\
\text { classes } \\
8 \% \text { Self-described } \\
\text { vegetarians }\end{array}$ & $\begin{array}{l}\text { Investigate the } \\
\text { prevalence and } \\
\text { characteristics of } \\
\text { vegetarian subjects } \\
\text { in the Women } \\
\text { Physician's Health } \\
\text { Study and } \\
\text { compare them } \\
\text { with the omnivore } \\
\text { cohort. }\end{array}$ & $\begin{array}{l}\text { No prior info } \\
\text { provided. }\end{array}$ & $\begin{array}{l}\text { Self-categorized } \\
\text { vegetarians were asked } \\
\text { why they were vegetarian. }\end{array}$ & $\begin{array}{l}32.1 \% \text { cited environmental } \\
\text { reasons. }\end{array}$ & n.a. \\
\hline
\end{tabular}

Notes n.a.: not assessed; $\mathrm{M}$ = arithmetic mean; $\mathrm{SD}$ = standard deviation; SES = socioeconomic status. *: As reported by [55]. 


\section{References}

1. Steinfeld, H.; Gerber, P.; Wassenaar, T.D.; Castel, V.; Rosales, M.; Rosales, M.; de Haan, C. Livestock's Long Shadow: Environmental Issues and Options; Food \& Agriculture Org.: Rome, Italy, 2006; ISBN 92-5-105571-8.

2. Slingo, J.M.; Challinor, A.J.; Hoskins, B.J.; Wheeler, T.R. Introduction: Food crops in a changing climate. Philos. Trans. R. Soc. B Biol. Sci. 2005, 360, 1983-1989. [CrossRef] [PubMed]

3. Bouwman, L.; Goldewijk, K.K.; Van Der Hoek, K.W.; Beusen, A.H.; Van Vuuren, D.P.; Willems, J.; Rufino, M.C.; Stehfest, E. Exploring global changes in nitrogen and phosphorus cycles in agriculture induced by livestock production over the 1900-2050 period. Proc. Natl. Acad. Sci. USA 2013, 110, 20882-20887. [PubMed]

4. Godfray, H.C.J.; Beddington, J.R.; Crute, I.R.; Haddad, L.; Lawrence, D.; Muir, J.F.; Pretty, J.; Robinson, S.; Thomas, S.M.; Toulmin, C. Food security: The challenge of feeding 9 billion people. Science 2010, 327, 812-818. [CrossRef] [PubMed]

5. Thornton, P.K. Livestock production: Recent trends, future prospects. Philos. Trans. R. Soc. B Biol. Sci. 2010, 365, 2853-2867. [CrossRef] [PubMed]

6. Dauvergne, P. The Shadows of Consumption: Consequences for the Global Environment; MIT Press: Cambridge, MA, USA, 2010; ISBN 0-262-26057-3.

7. Willett, W.; Rockström, J.; Loken, B.; Springmann, M.; Lang, T.; Vermeulen, S.; Garnett, T.; Tilman, D.; DeClerck, F.; Wood, A.; et al. Food in the Anthropocene: The EAT-Lancet Commission on healthy diets from sustainable food systems. Lancet 2019, 393, 447-492. [CrossRef]

8. Sabate, J.; Soret, S. Sustainability of plant-based diets: Back to the future. Am. J. Clin. Nutr. 2014, 100, 476S-482S. [CrossRef]

9. Glanz, K.; Rimer, B.K.; Viswanath, K. Health Behavior and Health Education: Theory, Research, and Practice; John Wiley \& Sons: Hoboken, NJ, USA, 2008; ISBN 0-470-43248-9.

10. Segovia-Siapco, G.; Sabaté, J. Health and sustainability outcomes of vegetarian dietary patterns: A revisit of the EPIC-Oxford and the Adventist Health Study-2 cohorts. Eur. J. Clin. Nutr. 2018. [CrossRef]

11. Ruby, M.B. Vegetarianism. A blossoming field of study. Appetite 2012, 58, 141-150. [CrossRef]

12. Kessler, C.S.; Holler, S.; Joy, S.; Dhruva, A.; Michalsen, A.; Dobos, G.; Cramer, H. Personality Profiles, Values and Empathy: Differences between Lacto-Ovo-Vegetarians and Vegans. Complement. Med. Res. 2016, 23, 95-102. [CrossRef]

13. Beardsworth, A.D.; Keil, E.T. Vegetarianism, Veganism, and Meat Avoidance: Recent Trends and Findings. Br. Food J. 1991, 93, 19-24. [CrossRef]

14. Fox, N.; Ward, K. Health, ethics and environment: A qualitative study of vegetarian motivations. Appetite 2008, 50, 422-429. [CrossRef] [PubMed]

15. Hussar, K.M.; Harris, P.L. Children who choose not to eat meat: A study of early moral decision-making. Soc. Dev. 2010, 19, 627-641. [CrossRef]

16. Jabs, J.; Devine, C.M.; Sobal, J. Model of the process of adopting vegetarian diets: Health vegetarians and ethical vegetarians. J. Nutr. Educ. 1998, 30, 196-202. [CrossRef]

17. Neale, R.J.; Tilston, C.H.; Gregson, K.; Stagg, T. Women vegetarians: Lifestyle considerations and attitudes to vegetarianism. Nutr. Food Sci. 1993, 93, 24-27. [CrossRef]

18. Dagevos, H.; Voordouw, J. Sustainability and meat consumption: Is reduction realistic? Sustain. Sci. Pract. Policy 2013, 9, 60-69. [CrossRef]

19. Moher, D.; Liberati, A.; Tetzlaff, J.; Altman, D.G.; The PRISMA Group. Preferred Reporting Items for Systematic Reviews and Meta-Analyses: The PRISMA Statement. PLoS Med. 2009, 6, e1000097. [CrossRef] [PubMed]

20. de Boer, J.; Schosler, H.; Boersema, J.J. Climate change and meat eating: An inconvenient couple? J. Environ. Psychol. 2013, 33, 1-8. [CrossRef]

21. De Groeve, B.; Bleys, B. Less Meat Initiatives at Ghent University: Assessing the Support among Students and How to Increase It. Sustainability 2017, 9, 1550. [CrossRef]

22. Pohjolainen, P.; Tapio, P.; Vinnari, M.; Jokinen, P.; Räsänen, P. Consumer consciousness on meat and the environment-Exploring differences. Appetite 2016, 101, 37-45. [CrossRef] [PubMed]

23. Vanhonacker, F.; Van Loo, E.J.; Gellynck, X.; Verbeke, W. Flemish consumer attitudes towards more sustainable food choices. Appetite 2013, 62, 7-16. [CrossRef] 
24. Graca, J.; Oliveira, A.; Calheiros, M.M. Meat, beyond the plate. Data-driven hypotheses for understanding consumer willingness to adopt a more plant-based diet. Appetite 2015, 90, 80-90. [CrossRef] [PubMed]

25. Cordts, A.; Nitzko, S.; Spiller, A. Consumer Response to Negative Information on Meat Consumption in Germany. Int. Food Agribus. Manag. Rev. 2014, 17, 83-106.

26. Truelove, H.B.; Parks, C. Perceptions of behaviors that cause and mitigate global warming and intentions to perform these behaviors. J. Environ. Psychol. 2012, 32, 246-259. [CrossRef]

27. Lea, E.; Worsley, A. Australian consumers' food-related environmental beliefs and behaviours. Appetite 2008, 50, 207-214. [CrossRef] [PubMed]

28. Clonan, A.; Wilson, P.; Swift, J.A.; Leibovici, D.G.; Holdsworth, M. Red and processed meat consumption and purchasing behaviours and attitudes: Impacts for human health, animal welfare and environmental sustainability. Public Health Nutr. 2015, 18, 2446-2456. [CrossRef] [PubMed]

29. Campbell-Arvai, V. Food-related environmental beliefs and behaviours among university undergraduates a mixed-methods study. Int. J. Sustain. High. Educ. 2015, 16, 279-295. [CrossRef]

30. de Boer, J.; de Witt, A.; Aiking, H. Help the climate, change your diet: A cross-sectional study on how to involve consumers in a transition to a low-carbon society. Appetite 2016, 98, 19-27. [CrossRef] [PubMed]

31. de Boer, J.; Schosler, H.; Aiking, H. "Meatless days" or "less but better"? Exploring strategies to adapt Western meat consumption to health and sustainability challenges. Appetite 2014, 76, 120-128. [CrossRef]

32. Siegrist, M.; Visschers, V.H.M.; Hartmann, C. Factors influencing changes in sustainability perception of various food behaviors: Results of a longitudinal study. Food Qual. Prefer. 2015, 46, 33-39. [CrossRef]

33. Tobler, C.; Visschers, V.H.M.; Siegrist, M. Eating green. Consumers' willingness to adopt ecological food consumption behaviors. Appetite 2011, 57, 674-682. [CrossRef]

34. Hunter, E.; Roos, E. Fear of climate change consequences and predictors of intentions to alter meat consumption. Food Policy 2016, 62, 151-160. [CrossRef]

35. European Commission. Brussels DG Communication COMM A1 'Research and Speechwriting' Flash Eurobarometer 367 (Attitudes of Europeans towards Building the Single Market for Green Products); European Commission: Brussels, Belgium, 2013.

36. Schösler, H.; de Boer, J.; Boersema, J.J.; Aiking, H. Meat and masculinity among young Chinese, Turkish and Dutch adults in the Netherlands. Appetite 2015, 89, 152-159. [CrossRef]

37. Graca, J.; Calheiros, M.M.; Oliveira, A. Attached to meat? (Un)Willingness and intentions to adopt a more plant-based diet. Appetite 2015, 95, 113-125. [CrossRef] [PubMed]

38. de Boer, J.; Aiking, H. Prospects for pro-environmental protein consumption in Europe: Cultural, culinary, economic and psychological factors. Appetite 2018, 121, 29-40. [CrossRef] [PubMed]

39. Hoffman, S.R.; Stallings, S.F.; Bessinger, R.C.; Brooks, G.T. Differences between health and ethical vegetarians. Strength of conviction, nutrition knowledge, dietary restriction, and duration of adherence. Appetite 2013, 65, 139-144. [CrossRef] [PubMed]

40. Dyett, P.A.; Sabate, J.; Haddad, E.; Rajaram, S.; Shavlik, D. Vegan lifestyle behaviors. An exploration of congruence with health-related beliefs and assessed health indices. Appetite 2013, 67, 119-124. [CrossRef] [PubMed]

41. Turner-McGrievy, G.M.; Moore, W.J.; Barr-Anderson, D. The Interconnectedness of Diet Choice and Distance Running: Results of the Research Understanding the Nutrition of Endurance Runners (RUNNER) Study. Int. J. Sport Nutr. Exerc. Metab. 2016, 26, 205-211. [CrossRef] [PubMed]

42. White, R.F.; Seymour, J.; Frank, E. Vegetarianism among us Women Physicians. J. Am. Diet. Assoc. 1999, 99, 595-598. [CrossRef]

43. Pribis, P.; Pencak, R.C.; Grajales, T. Beliefs and Attitudes toward Vegetarian Lifestyle across Generations. Nutrients 2010, 2, 523-531. [CrossRef] [PubMed]

44. Lindeman, M.; Sirelius, M. Food choice ideologies: The modern manifestations of normative and humanist views of the world. Appetite 2001, 37, 175-184. [CrossRef]

45. Rozin, P.; Markwith, M.; Stoess, C. Moralization and becoming a vegetarian: The transformation of preferences into values and the recruitment of disgust. Psychol. Sci. 1997, 8, 67-73. [CrossRef]

46. Schoesler, H.; de Boer, J.; Boersema, J.J. Fostering more sustainable food choices: Can Self-Determination Theory help? Food Qual. Prefer. 2014, 35, 59-69. [CrossRef]

47. Verain, M.C.; Dagevos, H.; Antonides, G. Sustainable food consumption. Product choice or curtailment? Appetite 2015, 91, 375-384. [CrossRef] [PubMed] 
48. Haverstock, K.; Forgays, D.K. To eat or not to eat. A comparison of current and former animal product limiters. Appetite 2012, 58, 1030-1036. [CrossRef] [PubMed]

49. De Backer, C.J.S.; Hudders, L. From Meatless Mondays to Meatless Sundays: Motivations for Meat Reduction among Vegetarians and Semi-vegetarians Who Mildly or Significantly Reduce Their Meat Intake. Ecol. Food Nutr. 2014, 53, 639-657. [CrossRef] [PubMed]

50. Peneau, S.; Fassier, P.; Alles, B.; Kesse-Guyot, E.; Hercberg, S.; Mejean, C. Dilemma between health and environmental motives when purchasing animal food products: Sociodemographic and nutritional characteristics of consumers. BMC Public Health 2017, 17, 876. [CrossRef] [PubMed]

51. Izmirli, S.; Phillips, C.J.C. The relationship between student consumption of animal products and attitudes to animals in Europe and Asia. Br. Food J. 2011, 113, 436-450. [CrossRef]

52. de Boer, J.; Schoesler, H.; Aiking, H. Towards a reduced meat diet: Mindset and motivation of young vegetarians, low, medium and high meat-eaters. Appetite 2017, 113, 387-397. [CrossRef]

53. Povey, R.; Wellens, B.; Conner, M. Attitudes towards following meat, vegetarian and vegan diets: An examination of the role of ambivalence. Appetite 2001, 37, 15-26. [CrossRef]

54. Beardsworth, A.; Keil, T. Health-related beliefs and dietary practices among vegetarians and vegans: A qualitative study. Health Educ. J. 1991, 50, 38-42. [CrossRef]

55. Hartmann, C.; Siegrist, M. Consumer perception and behaviour regarding sustainable protein consumption: A systematic review. Trends Food Sci. Technol. 2017, 61, 11-25. [CrossRef]

56. Lakoff, G. Why it matters how we frame the environment. Environ. Commun. 2010, 4, 70-81. [CrossRef]

57. Graham, T.; Abrahamse, W. Communicating the climate impacts of meat consumption: The effect of values and message framing. Glob. Environ. Chang.-Hum. Policy Dimens. 2017, 44, 98-108. [CrossRef]

58. Evans, L.; Milfont, T.L.; Lawrence, J. Considering local adaptation increases willingness to mitigate. Glob. Environ. Chang.-Hum. Policy Dimens. 2014, 25, 69-75. [CrossRef]

59. Feinberg, M.; Willer, R. Apocalypse soon? Dire messages reduce belief in global warming by contradicting just-world beliefs. Psychol. Sci. 2011, 22, 34-38. [CrossRef] [PubMed]

60. Howell, R.A. Investigating the long-term impacts of climate change communications on individuals' attitudes and behavior. Environ. Behav. 2014, 46, 70-101. [CrossRef]

61. Gussow, J.D.; Clancy, K.L. Dietary guidelines for sustainability. J. Nutr. Educ. 1986, 18, 1-5. [CrossRef]

62. Campbell, J.; Macdiarmid, J.I.; Douglas, F. Young people's perception of the environmental impact of food and their willingness to eat less meat for the sake of the environment: A qualitative study. Proc. Nutr. Soc. 2016, 75, E224. [CrossRef]

63. Macdiarmid, J.I.; Douglas, F.; Campbell, J. Eating like there's no tomorrow: Public awareness of the environmental impact of food and reluctance to eat less meat as part of a sustainable diet. Appetite 2016, 96, 487-493. [CrossRef]

64. Poulain, J.-P. The Sociology of Food: Eating and the Place of Food in Society; Bloomsbury Academic: London, UK; New York, NY, USA, 2017; ISBN 978-1-4725-8620-9.

(C) 2019 by the authors. Licensee MDPI, Basel, Switzerland. This article is an open access article distributed under the terms and conditions of the Creative Commons Attribution (CC BY) license (http://creativecommons.org/licenses/by/4.0/). 